https://helda.helsinki.fi

\title{
JIMWLK evolution of the odderon
}

Lappi, T.

2016-09-14

Lappi , T , Ramnath , A , Rummukainen , K \& Weigert , H 2016 , ' JIMWLK evolution of the odderon ' , Physical Review D , vol. 94 , no. 5 , 054014 . https://doi.org/10.1103/PhysRevD.94.054014

http://hdl.handle.net/10138/183650

https://doi.org/10.1103/PhysRevD.94.054014

cc_by

publishedVersion

Downloaded from Helda, University of Helsinki institutional repository.

This is an electronic reprint of the original article.

This reprint may differ from the original in pagination and typographic detail.

Please cite the original version. 
PHYSICAL REVIEW D 94, 054014 (2016)

\title{
JIMWLK evolution of the odderon
}

\author{
T. Lappi ${ }^{*}$ \\ Department of Physics, University of Jyväskylä, P.O. Box 35, Jyväskylä 40014, Finland \\ and Helsinki Institute of Physics, University of Helsinki, P.O. Box 64, \\ Helsinki 00014, Finland \\ A. Ramnath ${ }^{\dagger}$ \\ Department of Physics, University of Jyväskylä, P.O. Box 35, Jyväskylä 40014, Finland \\ K. Rummukainen \\ Department of Physics and Helsinki Institute of Physics, University of Helsinki, Helsinki 00014, Finland \\ H. Weigert ${ }^{\S}$ \\ Department of Physics, University of Cape Town, Private Bag X3, Rondebosch 7701, South Africa \\ (Received 21 June 2016; published 14 September 2016)

\begin{abstract}
We study the effects of a parity-odd "odderon" correlation in Jalilian-Marian-Iancu-McLerranWeigert-Leonidov-Kovner renormalization group evolution at high energy. Firstly we show that in the eikonal picture where the scattering is described by Wilson lines, one obtains a strict mathematical upper limit for the magnitude of the odderon amplitude compared to the parity-even Pomeron one. This limit increases with $N_{\mathrm{c}}$, approaching infinity in the infinite $N_{\mathrm{c}}$ limit. We use a systematic extension of the Gaussian approximation including both two- and three-point correlations which enables us to close the system of equations even at finite $N_{\mathrm{c}}$. In the large- $N_{\mathrm{c}}$ limit we recover an evolution equation derived earlier. By solving this equation numerically we confirm that the odderon amplitude decreases faster in the nonlinear case than in the linear Balitsky-Fadin-Kuraev-Lipatov limit. We also point out that, in the threepoint truncation at finite $N_{\mathrm{c}}$, the presence of an odderon component introduces azimuthal angular correlations $\sim \cos (n \varphi)$ at all $n$ in the target color field. These correlations could potentially have an effect on future studies of multiparticle angular correlations.
\end{abstract}

DOI: 10.1103/PhysRevD.94.054014

\section{INTRODUCTION}

High energy hadronic collisions at modern collider energies involve a dense system of gluons. At high enough energy the typical phase space density becomes nonperturbatively large, i.e. of the order of the inverse QCD coupling constant $1 / \alpha_{\mathrm{s}}$. In this limit it is better to describe these gluonic degrees of freedom as a classical color field than as a collection of individual particles, in what is known as the color glass condensate (CGC) picture [1,2]. In practice, the important degree of freedom here is the Wilson line, a path-ordered exponential in the color field. It gives the scattering amplitude of a colored high energy particle passing through the CGC target. Increasing the collision energy opens up phase space for the emission of even more gluons, which in this case are treated as quantum fluctuations on top of the classical field. These fluctuations can be systematically integrated out and included in the classical field. This procedure leads to renormalization

\footnotetext{
*tuomas.v.v.lappi@jyu.fi

†anramnat@student.jyu.fi

kari.rummukainen@helsinki.fi

\$heribert.weigert@uct.ac.za
}

group equations that describe the evolution of the Wilson lines as a function of collision energy.

The complete system of evolution equations is known as the Jalilian-Marian-Iancu-McLerran-Weigert-LeonidovKovner (JIMWLK) equation [3-13] or equivalently as the Balitsky hierarchy [14-17]. It describes the evolution of the whole probability distribution of Wilson lines. While this equation can be solved, at least at leading order, numerically [18-21], most phenomenological applications rely on simpler approximations. This is typically done by an evolution equation for an expectation value of Wilson lines that can be derived, in some approximation, from the equation for the full probability distribution. The usual approximation here is to use the large- $N_{\mathrm{c}}$ limit, which allows one to truncate the Balitsky hierarchy and obtain an evolution equation for the two-point function of Wilson lines known as the Balitsky-Kovchegov (BK) [14,22] equation. A related approximation, which has an identical dynamical content but can be used to construct the Wilson line expectation values at finite $N_{\mathrm{c}}$, is provided by the Gaussian approximation.

The Gaussian approximation relates all Wilson line correlators to a single two-point correlator. The purpose 
of this paper is to take the first step beyond the Gaussian approximation and introduce an intrinsic three-point correlation function of color charges using a method that can be extended to include all $n$-point functions up to any fixed finite number of points $m$ in what we refer to as an (exponential) $m$-point truncation. When this is used to evaluate the evolution equation for the two-point function of Wilson lines (the dipole operator), it turns out that the new three-point function only appears in a specific coordinate limit. It can, in fact, be rewritten as an imaginary part of the earlier two-point function. Physically this new degree of freedom corresponds to the odderon: an interaction by the exchange of a parity-odd particle. Similar modifications to the Gaussian average have been considered before in the context of the McLerran-Venugopalan (MV) [23-25] model (see e.g. [26-28]). Here, we will go beyond the work in these papers and derive evolution equations in rapidity for the odderon amplitude in the exponential threepoint approximation, extending earlier large- $N_{\mathrm{c}}$ results $[29,30]$ to finite $N_{\mathrm{c}}$. We will then numerically solve these evolution equations in a truncation in the harmonic number in the azimuthal direction. To determine the consistency of truncated JIMWLK evolution we complement our discussion with a numerical simulation of parity-even correlations using full untruncated JIMWLK evolution in the Langevin framework, reproducing the same qualitative behavior.

This paper is structured in the following way. First, in Sec. II, we motivate this study by an example of a phenomenological context in which the odderon amplitude appears directly. Then, in Sec. III, we point out that the origin of the dipole amplitude as a correlation function of Wilson lines that live on the SU(3) group manifold places stringent mathematical bounds on the size of the odderon. We then quantify these bounds for specific parametric forms of the initial conditions in Sec. IV. On the same basis we argue that, in the JIMWLK context, the odderon cannot affect observables that do not break rotational symmetry in the transverse plane. Section V presents the derivation of the evolution equations for the odderon component from an exponential $n$-point truncation. We solve these truncated equations in Sec. VI with a further approximation to the lowest nontrivial $\cos n \theta$ azimuthal harmonic. Then in Sec. VII we construct initial conditions for the JIMWLK equation that include an odderon component and study its evolution in a full (fixed coupling) JIMWLK simulation.

\section{OBSERVABLES AND CROSS SECTIONS}

The simplest application of JIMWLK evolution is calculating the total cross section in experiments like deep inelastic scattering (DIS), where a spacelike virtual photon is scattered on a nuclear target. At small $x$ and leading order in perturbation theory, the cross section is dominated by the $q \bar{q}$ component of the photon wave function, which interacts eikonally with the target. That is to say that the interaction is driven by an average of the dipole operator

$$
\hat{D}_{x, y}=\frac{1}{N_{\mathrm{c}}} \operatorname{tr}\left(U_{x} U_{y}^{\dagger}\right) .
$$

Using the diagrammatic notation introduced in [31] (see Fig. 1), the total cross section can be cast as

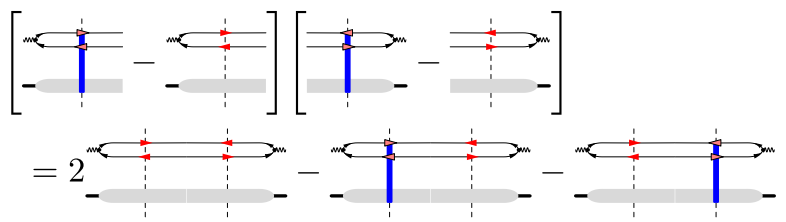

and allows access only to the real part of the dipole correlator contained in the last two terms of Eq. (2), since

$\left\langle\operatorname{tr}\left(U_{x} U_{y}^{\dagger}\right)\right\rangle(Y)+\left\langle\operatorname{tr}\left(U_{x}^{\dagger} U_{y}\right)\right\rangle(Y)=2\left\langle\operatorname{Retr}\left(U_{x} U_{y}^{\dagger}\right)\right\rangle(Y)$.

As indicated, the average will depend on $Y=(\ln 1 / x)$ with the $Y$ dependence governed by JIMWLK evolution.

The dipole operator does give rise to imaginary parts in a generic average $\left\langle\hat{D}_{x, y}\right\rangle(Y)$, i.e. over an ensemble not explicitly tailored to have a vanishing imaginary part, but one needs more detailed experiments to access this information. (We will argue in Sec. III that this is in fact an absolute statement, at least within the JIMWLK context.) The single transverse spin asymmetry (STSA) is such an
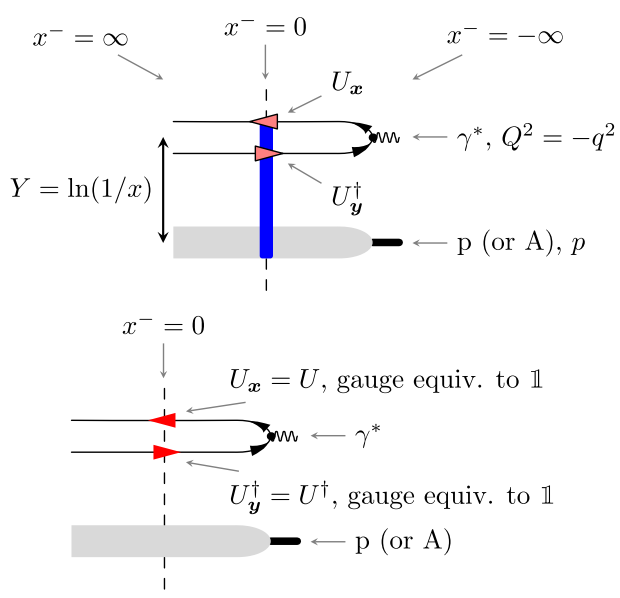

FIG. 1. Diagrammatic representation of the amplitude for $\gamma^{*} A$ scattering at small $x$ at momentum transfer $Q^{2}=-q^{2}$ as introduced in [31]. Light cone "time" $x^{-}$runs from right to left. The interacting "out state" (top diagram) contains nontrivial interactions between projectile and target. The interaction region is indicated by a vertical bar at $x^{-}=0$ with superimposed explicit markers for the Wilson lines picked up by each projectile constituent. An arrow to the left indicates a $U$, an arrow to the right a $U^{-1}$. Arrows on gluon lines stand for Wilson lines in the adjoint representation. The noninteracting "in state" (bottom diagram) instead has no interactions and correspondingly constant Wilson line factors at $x^{-}=0$ which are gauge equivalent to the unit element. 
observable. Kovchegov and Sievert [32] have in fact suggested a new mechanism to generate a contribution to STSA at small $x$ that is triggered by this imaginary part. The contribution suggested by Kovchegov and Sievert takes the diagrammatic form

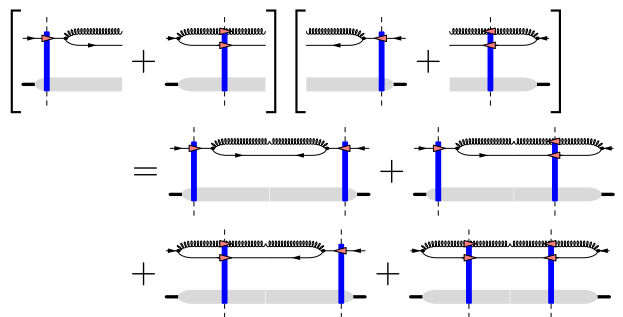

where the incoming quark is taken (probabilistically) from the incoming projectile.

In Eq. (4) the momentum and spin of the quark in the final state are tagged and the color in the initial state is summed over. Tagging the quark momentum leads to different coordinates on the corresponding Wilson lines in amplitude and complex conjugate amplitude. The gluon momentum is integrated over so that the gluon Wilson line in the last term cancels between the two sides of the cut. The color correlators from the right-hand side of Eq. (4) result in the following terms:

$$
\begin{aligned}
\mathcal{I}^{(q)}= & \left\langle\frac{\operatorname{tr}\left(U_{z} U_{\boldsymbol{y}}^{\dagger}\right)}{N_{\mathrm{c}}}-\frac{1}{d_{A}} \tilde{U}_{\boldsymbol{x}}^{a b} 2 \operatorname{tr}\left(t^{a} U_{z} t^{b} U_{\boldsymbol{w}}^{\dagger}\right)\right. \\
& \left.-\frac{1}{d_{A}} \tilde{U}_{\boldsymbol{x}}^{a b} 2 \operatorname{tr}\left(t^{a} U_{\boldsymbol{u}} t^{b} U_{\boldsymbol{y}}^{\dagger}\right)+\frac{\operatorname{tr}\left(U_{\boldsymbol{u}} U_{\boldsymbol{w}}^{\dagger}\right)}{N_{\mathrm{c}}}\right\rangle .
\end{aligned}
$$

The STSA is driven by the contributions that are antisymmetric under exchange of the quark and antiquark coordinates $z \leftrightarrow y$ and thus the imaginary part of, for example, the first term.

\section{GROUP THEORY CONSTRAINTS ON THE REAL AND IMAGINARY PARTS OF $q \bar{q}$ CORRELATORS}

Most readers familiar with JIMWLK and BK simulations will be prepared to accept that the $q \bar{q}$ and $q \bar{q} g$ correlators in Eq. (5), with normalization factors included, are real and interpolate between 1 at distances much smaller than the inverse saturation scale and 0 at pairwise separations much larger than the inverse saturation scale. This behavior is indeed respected by JIMWLK evolution in all its forms, provided it is satisfied by the initial condition.

This situation changes if one allows imaginary parts to arise. We will illustrate the situation with a discussion of the $q \bar{q}$ dipole correlator and its underlying configurations that appear explicitly in a Langevin simulation of JIMWLK evolution. To this end, note that these configurations appear as explicit $\mathrm{SU}\left(N_{\mathrm{c}}\right)$ matrices $U_{x} .{ }^{1}$ This remains true for the products entering the $q \bar{q}$ correlators: $U_{x} U_{y}^{\dagger} \in \mathrm{SU}\left(N_{\mathrm{c}}\right)$ is unitary and therefore has $N_{\mathrm{c}}$ eigenvalues of the form $e^{i \phi_{i}(\boldsymbol{x}, \boldsymbol{y})}, i=1, \ldots, N_{c} \in \mathbb{N}$. All of them are functions of both coordinates and live on the unit circle. The determinant condition $\operatorname{det}\left(U_{x} U_{y}^{\dagger}\right)=1$ then enforces that the phases of the eigenvalues sum to an integer multiple of $2 \pi$. Suppressing the coordinate dependence on the $\phi_{i}(\boldsymbol{x}, \boldsymbol{y})$ we have, for each pair of points

$1=\operatorname{det}\left(U_{\boldsymbol{x}} U_{\boldsymbol{y}}^{\dagger}\right)=e^{i \sum_{i=1}^{N_{\mathrm{c}}} \phi_{i}} \Leftrightarrow \sum_{i=1}^{N_{\mathrm{c}}} \phi_{i}=2 \pi n ; \quad n \in \mathbb{Z}$.

The trace of the dipole operator is therefore fully determined by $N_{\mathrm{c}}-1$ phases $\phi_{i} \in[0,2 \pi[$.

Using the constraint (6) to remove $\phi_{N_{\mathrm{c}}}$, one finds an expression for the trace of our group element $U_{x} U_{y}^{\dagger}$ that reads

$$
\frac{1}{N_{\mathrm{c}}} \operatorname{tr}\left(U_{\boldsymbol{x}} U_{\boldsymbol{y}}^{\dagger}\right)=\frac{1}{N_{\mathrm{c}}}\left(\sum_{i=1}^{N_{\mathrm{c}}-1} e^{i \phi_{i}}+e^{-i \sum_{i=1}^{N_{\mathrm{c}}-1} \phi_{i}}\right) .
$$

This trace falls into a simply connected closed subset of the complex plane, bounded by the curve

$$
h_{N_{\mathrm{c}}}(\theta)=\frac{1}{N_{\mathrm{c}}}\left(\left(N_{\mathrm{c}}-1\right) e^{i \theta}+e^{-i\left(N_{\mathrm{c}}-1\right) \theta}\right),
$$

where $\theta \in[0,2 \pi[$. (See [33] for a recent discussion of these textbook results.) Equation (8) has a very simple geometric interpretation: The center of a small circle (represented by the second term) is traveling along the perimeter of a large circle (represented by the first term). ${ }^{2}$ While the large circle is traversed once in a counterclockwise direction, the small traveling circle is traversed clockwise $N_{\mathrm{c}}$ times. The line traced out by $h_{N_{\mathrm{c}}}(\theta)$ in this manner is called a hypocycloid. The curve is fully contained in the unit circle and, for fixed $N_{\mathrm{c}}$, has cusps at the $N_{\mathrm{c}}$ th roots of unity-these are the only points where the curve touches the unit circle. These points correspond to specific group elements that form the center of the group $\left\{e^{i 2 \pi n / N_{\mathrm{c}}} \mathbb{1} \mid n \in \mathbb{Z} / N_{\mathrm{c}}\right\}$. In Fig. 2 both the geometric origin and the cusp structure are illustrated for a few values of $N_{\mathrm{c}}$. For $N_{\mathrm{c}} \rightarrow \infty$, the hypocycloid will approximate the unit circle. The value $N_{\mathrm{c}}=2$ does not allow for an imaginary part at all-the underlying reason is

\footnotetext{
${ }^{1}$ All arguments here assume that the coupling to the target is described fully through Wilson lines, i.e. the absence of subeikonal corrections, as is the case for all JIMWLK evolution.

${ }^{2}$ This description is adapted from the formula-alternatively, one might describe the boundary as the curve traced by a point on a circle of radius $1 / N_{\mathrm{c}}$ that rolls on the inside of the unit circle, starting with both circles touching at 1 .
} 

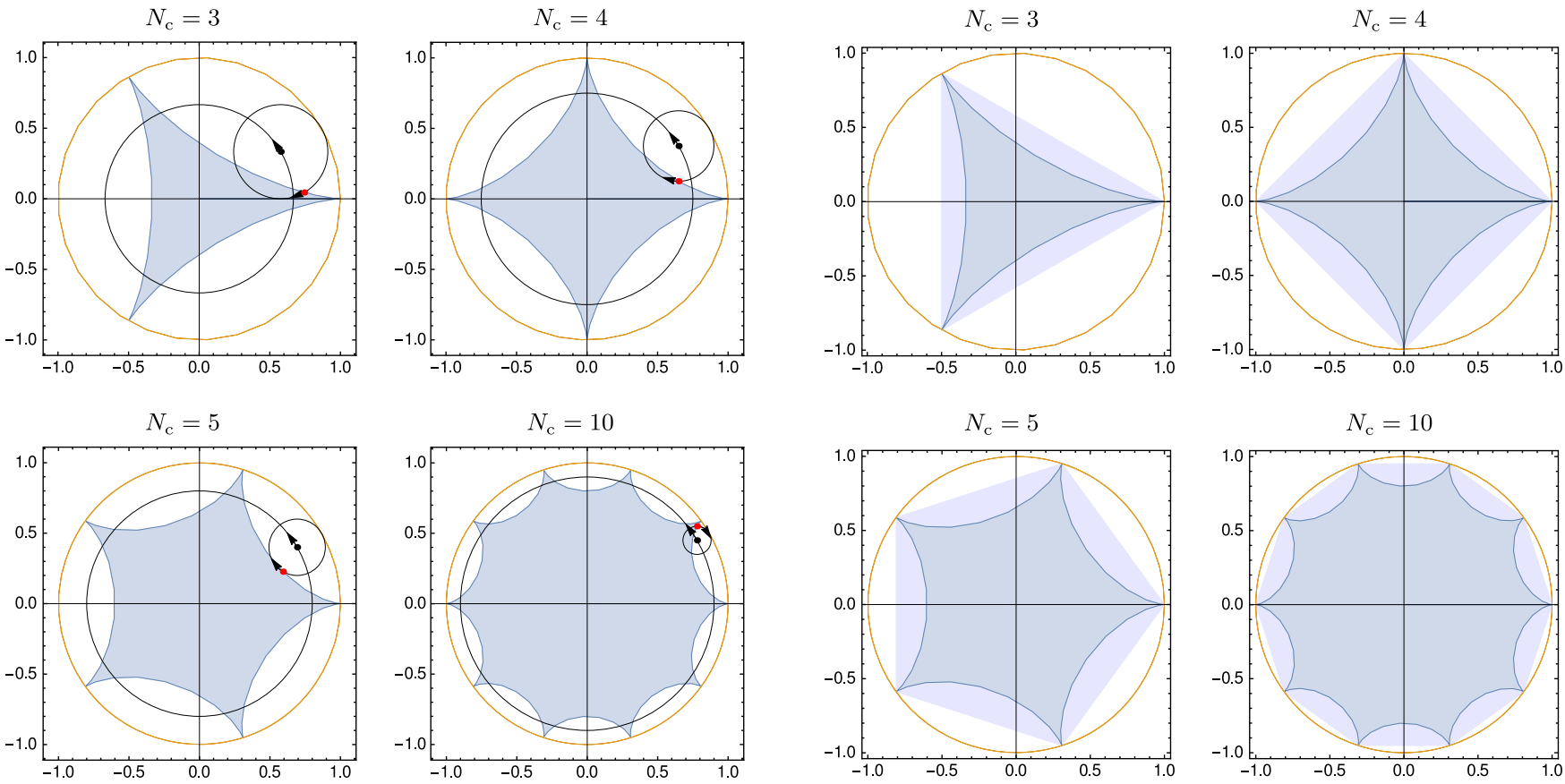

FIG. 2. Geometric origin and shape of the hypocycloids traced out by $h_{N_{\mathrm{c}}}(\theta)$. The shaded region corresponds to allowed values of $\operatorname{tr}\left(U_{x} U_{y}^{\dagger}\right) / N_{\mathrm{c}}$, i.e. the set of points in the complex plane reached by Eq. (7).

that the group is pseudoreal, i.e. $U^{\dagger}=\epsilon U \epsilon\left(\epsilon=\left[\epsilon^{i j}\right]\right)$ is isomorphic to $U$. In this vein, $N_{\mathrm{c}}=3$ is the first case that allows an imaginary part and has, at the same time, the strongest limitations on its size from the group structure alone.

The dipole correlator appears as an average over such configurations and can be parametrized in terms of two real degrees of freedom

$$
\begin{aligned}
S_{x y}(Y) & :=\left\langle\operatorname{tr}\left(U_{x} U_{y}^{\dagger}\right)\right\rangle(Y) / N_{\mathrm{c}} \\
& =1-P_{x y}(Y)+i O_{x y}(Y) \\
& =e^{-C_{\mathrm{F}}\left(\mathcal{P}_{x y}+i \mathcal{O}_{x y}\right)(Y)},
\end{aligned}
$$

i.e. either directly through real and imaginary parts $\left(1-P_{x y}\right.$ and $O_{x y}$, respectively) or exponentially via a logarithmic modulus and phase $\left(\mathcal{P}_{x y}\right.$ and $\mathcal{O}_{x y}$, respectively). Noting that complex conjugation simply swaps the coordinates on $S$, $S_{x y}^{*}=S_{y x}$ implies that $P$ and $\mathcal{P}$ are symmetric, while $O$ and $\mathcal{O}$ are antisymmetric under the exchange of $\boldsymbol{x}$ and $\boldsymbol{y}$. This symmetry property links them to the Pomeron and odderon, respectively.

One striking observation is that nothing inherently prevents the real part, the Pomeron contribution, from taking negative values - the hypocycloids allow negative real parts. In fact the Wilson line dipole correlators of Eq. (9) are averages of configurations falling into the interior of the hypocycloid $h_{N_{\mathrm{c}}}(\theta)$ and thus are even
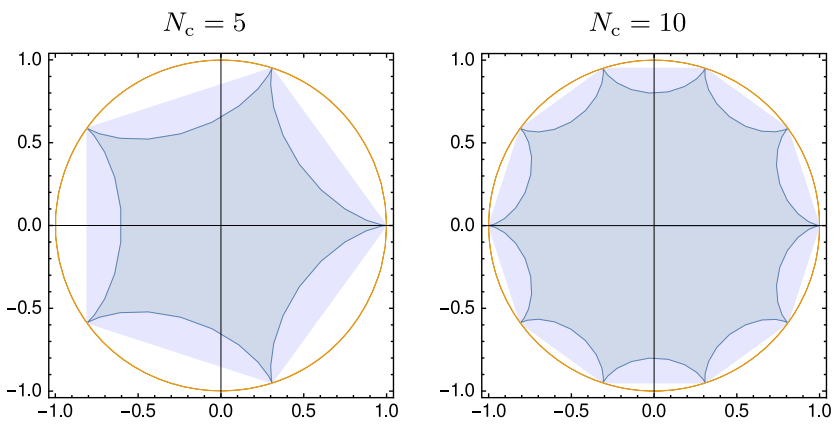

FIG. 3. Average group element traces must fall within a polygon connecting the $N_{\mathrm{c}}$ th roots of unity. For $N_{\mathrm{c}} \rightarrow \infty$ the polygon will approximate the unit circle.

slightly less constrained: Such an average may fall outside the hypocycloid but must remain inside a polygon connecting the cusps, as illustrated for a few values of $N_{\mathrm{c}}$ in Fig. 3. For $N_{c} \in \mathbb{Z} \geq 2$, where there is a well-defined interior, the bounding polygon can be parametrized by

$$
p_{N_{c}}(\theta)=\frac{\cos \left(\frac{\pi}{N_{c}}\right)}{\cos \left(\bmod \left(\theta, \frac{2 \pi}{N_{c}}\right)-\frac{\pi}{N_{c}}\right)} e^{i \theta},
$$

again with $\theta \in[0,2 \pi[$.

Let us emphasize that this is not in contradiction with the bounds observed for real correlators in earlier simulations. In fact, consistent, real-valued initial conditions for the $q \bar{q}$ and $q \bar{q} g$ correlators of Eq. (5) during evolution lie between the fixed points at 1 and 0 and the evolution equation does not develop an imaginary part starting from a real initial condition respecting these bounds.

This behavior is in fact a generic requirement on a consistent evolution equation for Wilson line dipole correlators. To expose the physics content of this statement, parametrize a point inside the hypocycloid or inside its bounding polygon by a real factor $\rho \in[0,1]$ and a point on the boundary $b_{N_{c}}(\theta)$ [with $b_{N_{c}}(\theta)$ either given by $h_{N_{c}}(\theta)$ or $p_{N_{c}}(\theta)$ ], so that the Wilson line correlator (the average) takes the form

$$
\left\langle\operatorname{tr}\left(U_{x} U_{y}^{\dagger}\right)\right\rangle(Y) / N_{\mathrm{c}}=\rho_{x y}(Y) b_{N_{\mathrm{c}}}\left(\theta_{x y}(Y)\right) .
$$

Like for the ingredients of Eq. (9) there are clear symmetry requirements on $\rho$ and $\theta$ in Eq. (11): They must be 
symmetric and antisymmetric, respectively, under exchange of $\boldsymbol{x}$ and $\boldsymbol{y}$. If the measurement is both translationally and rotationally symmetric in the transverse plane [i.e. in the absence of an additional transverse vector $\hat{s}$ to furnish the sign change via a factor $\hat{\boldsymbol{s}} \cdot(\boldsymbol{x}-\boldsymbol{y})]$, the contribution of any antisymmetric function such as $\theta_{x y}$ must vanish. In that case Eq. (11) can be reduced to $S_{x y}(Y)=\rho_{x y}(Y) \in[0,1]$; i.e. the solutions are restricted to the intersection of the hypocycloid with the positive real axis. (See also Sec. VII where this mechanism is demonstrated for JIMWLK ensembles.)

The need for additional directional information is a physics requirement: To be able to see an odderon contribution in an experiment one needs to break rotational symmetry in the transverse plane such as is done by measuring a spin asymmetry in STSA. The total cross section, by contrast, averages over all directions $\hat{\boldsymbol{s}}$ (in the average that forms the correlator) and thus forces $b_{N_{c}} \rightarrow 1$. In this case there is no scope for an average odderon contribution to couple to the real part visible in Eq. (3); it is zero throughout.

As a mathematical constraint, one needs an imaginary part in the initial condition for the average to allow it to move away from the interval $[0,1]$ and, in particular, for the real part to turn negative. (Note that this does not imply that individual configurations may not fall onto the negative real axis; see again Sec. VII for explicit examples.)

To conclude this section, we note that the perturbative limit $r \rightarrow 0$ takes $\left\langle\operatorname{tr}\left(U_{x} U_{y}^{\dagger}\right)\right\rangle(Y) / N_{\mathrm{c}} \rightarrow 1$ and thus is associated with strong correlations and the trivial center element $U_{x} U_{x}^{\dagger}=1$. The origin on the other hand corresponds to $\left\langle\operatorname{tr}\left(U_{x} U_{y}^{\dagger}\right)\right\rangle(Y) / N_{\mathrm{c}} \rightarrow 0$ and thus the long distance limit where the Wilson lines are uncorrelated. The remaining center elements (at least for $N_{c}=3$ ) correspond to maximally anticorrelated configurations.

If such configurations are not present with considerable weight, the averages will not have any chance of being pulled outside the hypocycloid into the remainder of then enveloping polygon. The perturbatively motivated initial conditions discussed in Sec. IV below do not lead to such behavior despite the presence of noticeable anticorrelation in one of the examples.

\section{CONSTRAINTS ON THE INITIAL CONDITIONS}

The physics expectations for the total cross section in the absence of an imaginary part severely restrict the form of the initial condition. The simplest assumption, based on exponentiating the $\boldsymbol{r}^{2}$ behavior of leading-order light cone perturbation theory, leads to the well-known GolecBiernat-Wüsthoff (GBW) [34] ansatz $S_{x y}=e^{-(x-y)^{2} Q_{s}^{2} / 4}$, where $Q_{\mathrm{s}}$ is the GBW saturation scale. The MV model modifies this with a logarithmic correction in the exponent, and evolution at leading order will carry any of these into a scaling form entirely imposed by the nonlinear form of the evolution equation. For our discussion here, all of these forms are suitable since at leading order (see, however [35-38]) evolution will quickly readjust these to take on the features of the scaling form.

If we allow for a nonzero odderon admixture, the choice of a physically meaningful initial condition for the pair of $\mathcal{P}$ and $\mathcal{O}$ needs some thought. At short distance, light cone perturbation theory leads to $\mathcal{P}_{x y} \propto|\boldsymbol{x}-\boldsymbol{y}|^{2}$ and $\mathcal{O}_{x y} \propto|\boldsymbol{x}-\boldsymbol{y}|^{3}$ [29], but the symmetry properties imposed by complex conjugation require the presence of an additional transverse vector $\hat{\boldsymbol{s}}$ as discussed in Sec. III. We thus expect a short distance $r \ll 1 / Q_{\mathrm{s}}$ behavior of the form

$$
\mathcal{P}_{x y} \propto \boldsymbol{r}^{2}, \quad \mathcal{O}_{x y} \propto \kappa \boldsymbol{r}^{2} \boldsymbol{r} \cdot \hat{\boldsymbol{s}}=\kappa r^{3} \hat{\boldsymbol{r}} \cdot \hat{\boldsymbol{s}},
$$

where $\boldsymbol{r}=\boldsymbol{x}-\boldsymbol{y}$ and $\hat{\boldsymbol{r}}=\boldsymbol{r} / r$. If we measure both contributions in (12) in the same units, $\kappa$ serves to parametrize the normalization of the odderon relative to the Pomeron amplitude. We will see in the following that it is constrained by the group theory limits on the scattering amplitude in the dilute limit $r \rightarrow 0$.

As discussed in Sec. III, we can impose the group theory constraints on the amplitude at two levels of rigor. Physically we would expect that the average amplitude itself is within the group manifold. In principle, it is also possible that the average amplitude is within the polygon region defined by linear combinations of amplitudes in the group.

In the first, more physical case, this leads in the $r \rightarrow 0$ limit to the constraint

$$
\mathcal{O}_{r} \leq \frac{\sqrt{2}}{3} \frac{N_{\mathrm{c}}-2}{\sqrt{N_{\mathrm{c}}-1}}\left(\mathcal{P}_{r}\right)^{3 / 2}
$$

Assuming that the Pomeron has the perturbative behavior

$$
\mathcal{P}_{r} \approx r^{2} Q_{\mathrm{s}}^{2} / 4
$$

and parametrizing the odderon with a general power law as

$$
\mathcal{O}_{r} \approx \kappa\left(r Q_{\mathrm{s}} / 2\right)^{\alpha},
$$

this leads to the constraint $\alpha \geq 3$. Thus, the result $\mathcal{O}_{r} \sim r^{3}$ of Ref. [29] indeed corresponds to the mildest $r$ dependence allowed by the group theory constraint. Assuming now the power $\alpha=3$ we get the limit

$$
\kappa \leq \frac{\sqrt{2}}{3} \frac{N_{\mathrm{c}}-2}{\sqrt{N_{\mathrm{c}}-1}} \underset{N_{\mathrm{c}}=3}{=} \frac{1}{3} .
$$

We want to stress the remarkable nature of this result. With linear Balitsky-Fadin-Kuraev-Lipatov (BFKL) evolution the magnitudes of the Pomeron and odderon amplitudes are only limited by phenomenology. The interpretation of 
the scattering amplitude in terms of the Wilson line immediately places a nonperturbative mathematical upper limit on the odderon amplitude.

Strictly speaking the averages of $\mathrm{SU}\left(N_{\mathrm{c}}\right)$ matrices need not be inside the group. Thus, in principle the upper limit for the odderon follows from restricting the expectation value of the amplitude to lie inside the $N_{\mathrm{c}}$-sided polygon with corners at the $N_{\mathrm{c}}$ roots of unity. This leads in the limit $r \rightarrow 0$ to

$$
\mathcal{O}_{r} \leq \frac{\sin \frac{2 \pi}{N_{\mathrm{c}}}}{1-\cos \frac{2 \pi}{N_{\mathrm{c}}}} \mathcal{P}_{\boldsymbol{r}}
$$

For amplitudes parametrized as in (14) and (15), this leads to the less stringent limit $\alpha \geq 2$. For the limiting power $\alpha=2$ the odderon amplitude is constrained to $\kappa(\alpha=2) \leq \frac{\sin \frac{2 \pi}{N_{\mathrm{c}}}}{1-\cos \frac{2 \pi}{N_{\mathrm{c}}}}$. For the value $\alpha=3$, any value of $\kappa$ satisfies this more lax version of the group theory constraint sufficiently close to $r=0$. From larger dipole sizes one does obtain an upper limit on $\kappa$, but this limit is universal in the same way; i.e. it depends on the functional form at larger $r$.

As practical initial conditions for evolution including the odderon, we suggest two possible extensions of the GBW parametrization:

$$
\left\langle\operatorname{tr}\left(U_{x} U_{y}^{\dagger}\right)\right\rangle\left(Y_{0}\right) / N_{\mathrm{c}}=e^{-r^{2} Q_{0}^{2} / 4+i \kappa r^{3} Q_{0}^{3} / 8 \hat{r} \cdot \hat{s}}
$$

or

$$
\left\langle\operatorname{tr}\left(U_{x} U_{\boldsymbol{y}}^{\dagger}\right)\right\rangle\left(Y_{0}\right) / N_{\mathrm{c}}=e^{-r^{2} Q_{0}^{2} / 4}\left(1+i \kappa r^{3} Q_{0}^{3} / 8 \hat{\boldsymbol{r}} \cdot \hat{\boldsymbol{s}}\right) .
$$

Exponentiating the real part ensures that both the short- and long-distance behavior are qualitatively correct: The $\boldsymbol{x}-\boldsymbol{y} \rightarrow \mathbf{0}$ limit produces 1 and the limit $\boldsymbol{x}-\boldsymbol{y} \rightarrow \infty$ produces 0 . We have no similar bias for or against exponentiating the imaginary contribution, but the two choices have very different consequences: Ansatz (18) leads to anticorrelations in the real part while (19) does not-see Figs. 4 and 5. This is qualitatively different, but neither option can be excluded on purely theoretical grounds.

The initial conditions (18) and (19) are visualized in Fig. 6. As discussed above, in order for the average amplitude to stay within the group manifold, we must have $\kappa \leq 1 / 3$. For the functional form of Eq. (19) the parametrization stays within the triangle allowed for linear combinations of $\mathrm{SU}\left(N_{\mathrm{c}}=3\right)$ Wilson lines for

$$
\kappa \leq \frac{2 \sqrt{\frac{2}{3}}\left(e^{W\left(-3 e^{-3 / 2} / 2\right)+3 / 2}\right)}{\left(3+2 W\left(-3 e^{-3 / 2} / 2\right)\right)^{3 / 2}} \approx 0.9867,
$$

where $W$ is the Lambert function, defined as the solution of $z=W(z) e^{W(z)}$. We see that for the other parametrization
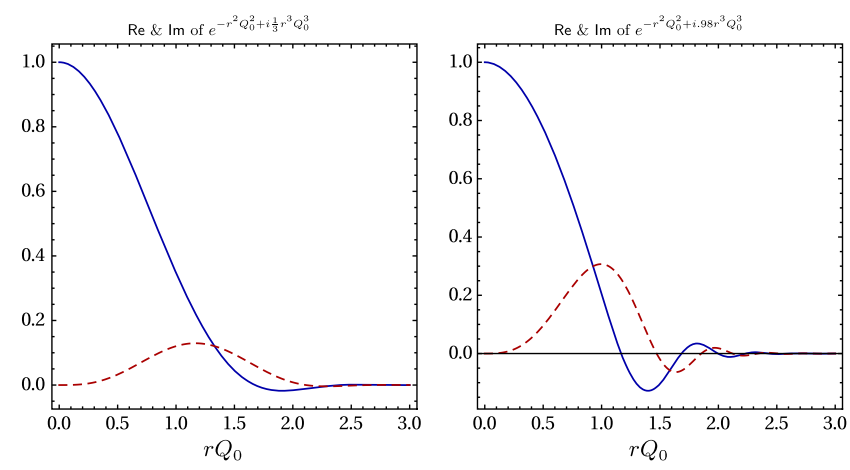

FIG. 4. Perturbatively motivated initial conditions for both real and imaginary parts (solid blue and red dashed curve, respectively) of $\left\langle\operatorname{tr}\left(U_{\boldsymbol{x}} U_{\boldsymbol{y}}^{\dagger}\right)\right\rangle\left(Y_{0}\right) / N_{\mathrm{c}}=e^{-r^{2} Q_{0}^{2}+i \kappa r^{3} Q_{0}^{3} \hat{\boldsymbol{r}} \cdot \hat{\boldsymbol{s}}}$ at $\hat{\boldsymbol{r}} \cdot \hat{\boldsymbol{s}}=1$ assuming no extreme anticorrelations to drive the correlator outside the hypocycloid (left) and relaxing this condition (right). In both cases, real and imaginary parts show modulation only near $Q_{s}\left(Y_{0}\right)$.
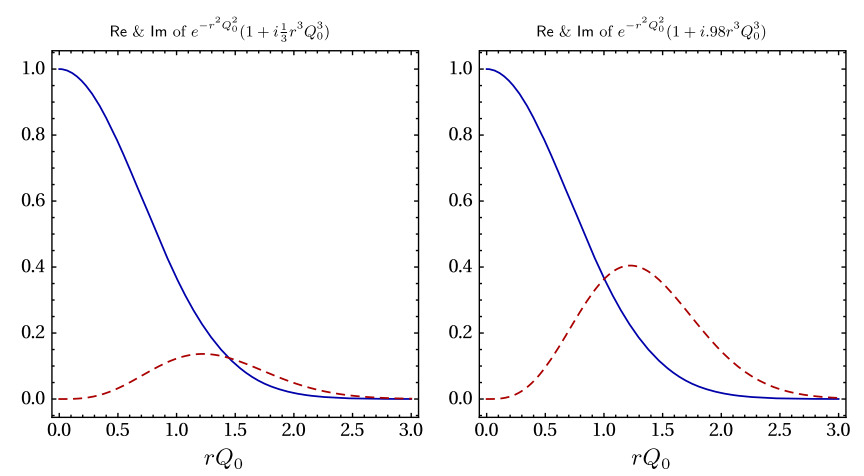

FIG. 5. Perturbatively motivated initial conditions for both real and imaginary parts (solid blue and red dashed curve, respectively) of $\left\langle\operatorname{tr}\left(U_{\boldsymbol{x}} U_{\boldsymbol{y}}^{\dagger}\right)\right\rangle\left(Y_{0}\right) / N_{\mathrm{c}}=e^{-r^{2} Q_{0}^{2}+i \kappa r^{3} Q_{0}^{3} \hat{r} \cdot \hat{s}}$ at $\hat{\boldsymbol{r}} \cdot \hat{\boldsymbol{s}}=1$ assuming no extreme anticorrelations to drive the correlator outside the hypocycloid (left) and relaxing this condition (right). In both cases real and imaginary parts show modulation only near $Q_{s}\left(Y_{0}\right)$.

(18), the amplitude stays within the triangular region for the same values $\kappa$.

In both cases, $\kappa \lesssim 1$ so that real and imaginary parts show modulation only near $Q_{s}\left(Y_{0}\right)$ and thus provide perturbatively consistent starting points for evolution in a calculation where $Q_{s}\left(Y_{0}\right)$ is assumed to be in the perturbative domain. Note that the size of the odderon peak (the maximum of imaginary parts shown in red in Figs. 4 and 5) is severely limited by the bounds on $\kappa$.

\section{GAUSSIAN AND HIGHER-ORDER EXPONENTIAL TRUNCATIONS}

The dependence of the Wilson lines on the factorization rapidity that separates the small- and large- $x$ degrees of freedom in the CGC formalism is given by the JIMWLK equation [3-13] or equivalently by the Balitsky hierarchy 

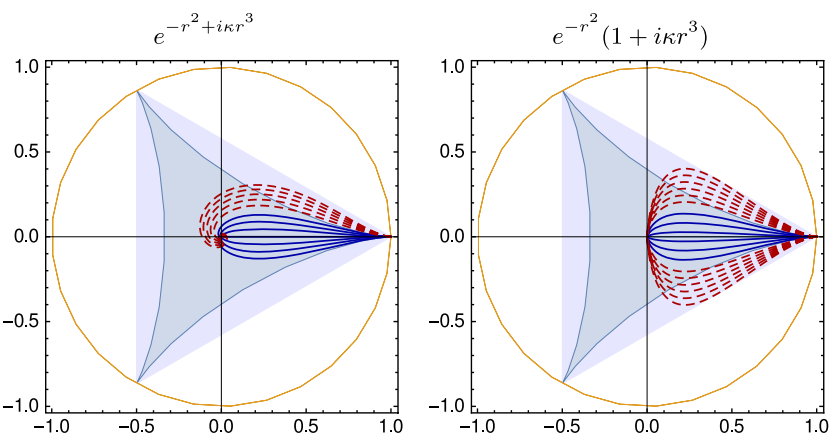

FIG. 6. Perturbatively motivated initial conditions for Pomeron + odderon configuration averages. Lowest-order perturbative calculations yield $r^{2}$ and $r^{3}$ behavior for small $r=|\boldsymbol{x}-\boldsymbol{y}|$ in the Pomeron and odderon channel, respectively. The plots show how different initial conditions seeded with this behavior at small $r$ traverse the complex plane. In the parametrizations shown $\kappa$ parametrizes the ratio of characteristic scales in the Pomeron and odderon sector. To fall into the allowed region $|\kappa|$ must be small $\left(|\kappa| \leq \frac{1}{3}\right.$, blue; $\kappa \leq .98$, dashed red and blue lines), which limits the possible size of the Pomeron contribution in the initial condition; see Figs. 4 and 5.

[14-17]. The Balitsky hierarchy is a coupled set of integrodifferential equations for operators made from products of Wilson lines. The first equation of the hierarchy is

$$
\begin{aligned}
\frac{\mathrm{d}}{\mathrm{d} Y}\left\langle\operatorname{tr}\left(U_{x} U_{\boldsymbol{y}}^{\dagger}\right)\right\rangle= & \frac{\alpha_{\mathrm{s}}}{\pi^{2}} \int \mathrm{d}^{2} z \tilde{\mathcal{K}}_{x z y} \\
& \times\left\langle\tilde{U}_{z}^{a b} \operatorname{tr}\left(t^{a} U_{x} t^{b} U_{y}^{\dagger}\right)-C_{\mathrm{F}} \operatorname{tr}\left(U_{x} U_{y}^{\dagger}\right)\right\rangle,
\end{aligned}
$$

where $\tilde{\mathcal{K}}_{x z y}=\frac{(x-y)^{2}}{(x-z)^{2}(z-y)^{2}}$. It relates the rapidity dependence of the dipole operator to a combination of dipole and threepoint operators. The equation is derived by considering the emission of one soft gluon from the dipole; it is therefore not surprising that the three-point function involved is the same as in the STSA cross section (5). Using the Fierz identity, Eq. (21) becomes

$$
\begin{aligned}
\frac{\mathrm{d}}{\mathrm{d} Y}\left\langle\frac{\operatorname{tr}\left(U_{x} U_{y}^{\dagger}\right)}{N_{\mathrm{c}}}\right\rangle= & \frac{\alpha_{\mathrm{s}}}{\pi^{2}} \frac{N_{\mathrm{c}}}{2} \int \mathrm{d}^{2} z \tilde{\mathcal{K}}_{x z y} \\
& \times\left\langle\frac{\operatorname{tr}\left(U_{x} U_{y}^{\dagger}\right)}{N_{\mathrm{c}}} \frac{\operatorname{tr}\left(U_{x} U_{y}^{\dagger}\right)}{N_{\mathrm{c}}}-\frac{\operatorname{tr}\left(U_{x} U_{y}^{\dagger}\right)}{N_{\mathrm{c}}}\right\rangle .
\end{aligned}
$$

This form is often used to truncate the hierarchy by factorizing

$$
\left\langle\frac{\operatorname{tr}\left(U_{x} U_{y}^{\dagger}\right)}{N_{\mathrm{c}}} \frac{\operatorname{tr}\left(U_{x} U_{y}^{\dagger}\right)}{N_{\mathrm{c}}}\right\rangle \stackrel{\text { fact }}{\longrightarrow}\left\langle\frac{\operatorname{tr}\left(U_{x} U_{y}^{\dagger}\right)}{N_{\mathrm{c}}}\right\rangle\left\langle\frac{\operatorname{tr}\left(U_{x} U_{y}^{\dagger}\right)}{N_{\mathrm{c}}}\right\rangle
$$

in the spirit of an independent scattering approximation for dipoles. The resulting closed mean-field equation

$$
\begin{aligned}
& \frac{\mathrm{d}}{\mathrm{d} Y}\left\langle\frac{\operatorname{tr}\left(U_{x} U_{y}^{\dagger}\right)}{N_{\mathrm{c}}}\right\rangle=\frac{\alpha_{\mathrm{s}}}{\pi^{2}} \frac{N_{\mathrm{c}}}{2} \int \mathrm{d}^{2} z \tilde{\mathcal{K}}_{x z y} \\
& \quad \times\left[\left\langle\frac{\operatorname{tr}\left(U_{x} U_{y}^{\dagger}\right)}{N_{\mathrm{c}}}\right\rangle\left\langle\frac{\operatorname{tr}\left(U_{x} U_{y}^{\dagger}\right)}{N_{\mathrm{c}}}\right\rangle-\left\langle\frac{\operatorname{tr}\left(U_{x} U_{y}^{\dagger}\right)}{N_{\mathrm{c}}}\right\rangle\right]
\end{aligned}
$$

is the Balitsky-Kovchegov equation [14,22] and is a crucial tool in practical phenomenological applications of the CGC formalism.

To develop a better understanding of the possible physics content of the correlators in Eq. (5) and their JIMWLK evolution, we now turn to a set of systematically extendable truncations of the Balitsky hierarchy associated with the dipole operator, the simplest of which is known as the Gaussian truncation.

The motivation and prototype of this truncation is a procedure applied to the calculation of the gluon density in the MV model already in Ref. [3] and heavily reused since [31,39-43]. The method relies on the fact that Wilson lines are given as path-ordered exponentials in the gauge field as

$$
U_{\boldsymbol{x}}=P \exp \left\{i g \int \mathrm{d} x^{-} A_{\boldsymbol{x}, x^{-}}^{a,+}\right\}
$$

and the assumption-intrinsic to the MV model — that the correlators of the $A$ field in the exponent obey Gaussian statistics with only a local correlation in the longitudinal coordinate

$$
g^{2}\left\langle A_{\boldsymbol{x}, x^{-}}^{a,+} A_{\boldsymbol{y}, y^{-}}^{b,+}\right\rangle=\delta\left(x^{-}-y^{-}\right) \delta^{a b} G_{x^{-}, x y} .
$$

The MV model further assumes a specific form of the correlation function

$$
\partial_{x}^{2} \partial_{y}^{2} G_{x^{-}, x y}=g^{2} \mu^{2}\left(x^{-}\right) \delta_{x y},
$$

but the latter is not a necessary ingredient for the truncations and will not be assumed in the following.

One advantage of such a procedure is that all possible correlators with any number of Wilson lines automatically obey all group theoretical relations imposed in any possible coincidence limit, such as the relationships listed in Fig. 7. More generally, we can achieve this feature by parametrizing Wilson line correlators in a Gaussian manner via

$$
\langle\ldots\rangle(\eta)=\left\langle P_{\eta} e^{\int_{\eta_{0}}^{\eta} \mathrm{d} \eta^{\prime}\left[\frac{1}{2} \int_{u v} G_{u v}\left(\eta^{\prime}\right) i \nabla_{u}^{a} i \nabla_{v}^{a}\right]} \ldots\right\rangle\left(\eta_{0}\right),
$$

where we have replaced $x^{-}$by a general coordinate space longitudinal (rapidity) variable $\eta \sim \ln x^{-}$. Practically, Eq. (28) allows one to find a parametrization for any set of correlators with consistent coincidence limits (such as listed in Fig. 7) by solving the functional differential equation

$$
\frac{\mathrm{d}}{\mathrm{d} \eta}\langle F[U]\rangle(\eta)=\frac{1}{2} \int_{\boldsymbol{u}, \boldsymbol{v}}\left\langle G_{\boldsymbol{u} \boldsymbol{v}}(X) i \nabla_{u}^{a} i \nabla_{\boldsymbol{v}}^{a} F[U]\right\rangle(\eta)
$$




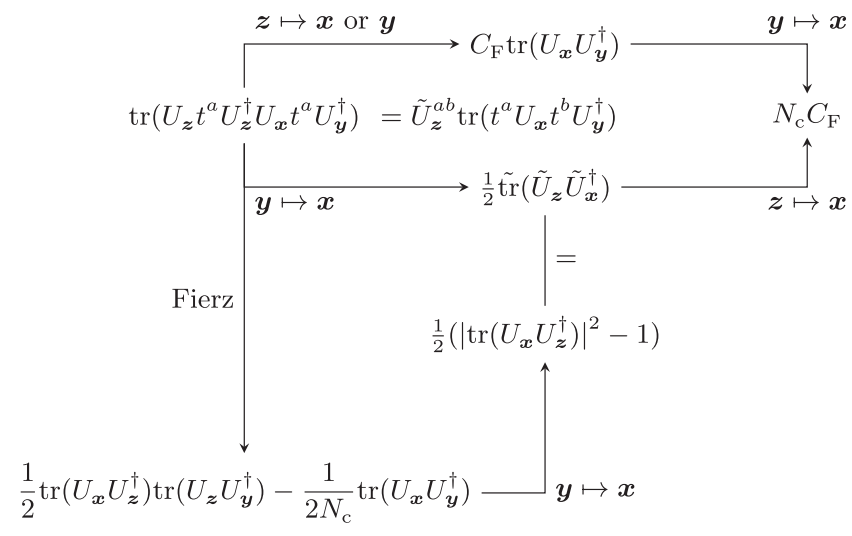

FIG. 7. Correlator relations between $q \bar{q} g, q \bar{q}$ and $g g$. to parametrize some singlet observable $\langle F[U]\rangle$ in terms of the two-point correlator $G_{u v}$. The simplest example is the $q \bar{q}$ correlator with $F[U] \rightarrow \operatorname{tr}\left(U_{x} U_{y}^{\dagger}\right) / N_{c}$ for which this functional equation indeed turns into a closed differential equation. For more complicated operators such as $q^{2} \bar{q}^{2}$, the functional equation turns into a set of coupled differential equations that need to be solved simultaneously. The expectation values of several different Wilson line operators with a Gaussian weight have been calculated in the literature, e.g. in Refs. [3,31,40-45].

The simplest generalization of the Gaussian truncation is what we will call the three-point exponential truncation. It includes the two-point contribution from the Gaussian truncation but adds all mathematically independent threepoint functions to the exponential. This can be summarized by

$$
\begin{aligned}
\langle\ldots\rangle(\eta)= & \left\langleP _ { \eta } \operatorname { e x p } \left\{\int _ { \eta _ { 0 } } ^ { \eta } \mathrm { d } \eta ^ { \prime } \left[\frac{1}{2} \int_{\boldsymbol{u} v} G_{\boldsymbol{u} v}\left(\eta^{\prime}\right) i \nabla_{u}^{a} i \nabla_{v}^{a} \frac{1}{3 !} \int_{\boldsymbol{u v w}} G_{u v w}^{d}\left(\eta^{\prime}\right) d^{a b c} i \nabla_{u}^{a} i \nabla_{v}^{b} i \nabla_{w}^{c}\right.\right.\right. \\
& \left.\left.\left.\times \frac{1}{3 !} \int_{\boldsymbol{u} v \boldsymbol{w}} G_{u v w}^{f}\left(\eta^{\prime}\right) f^{a b c} i \nabla_{u}^{a} i \nabla_{v}^{b} i \nabla_{\boldsymbol{w}}^{c}+4 \mathrm{pts}\right]\right\} \ldots\left(\eta_{0}\right)\right\rangle .
\end{aligned}
$$

As indicated there is, in principle, no obstruction to higher $n$-point functions to this parametrization functional.

Note that in Eq. (30) both $G$ and $G^{d}$ are fully symmetrical under exchange of any pair of transverse coordinates, while $G^{f}$ has slightly more complicated features but will not enter any of the correlators in Eqs. (3) and (5) so that we have no need to discuss it in any more detail.

We choose $G_{u \boldsymbol{u}}=0$ and $G_{u u u}=0$ as this simplifies some of the expressions below from the outset. The correlators in Eq. (21) then take the form

$$
S_{x y}=\frac{\left\langle\operatorname{tr}\left(U_{x} U_{y}^{\dagger}\right)\right\rangle}{N_{\mathrm{c}}}=e^{-C_{\mathrm{F}} \mathcal{G}_{x y}}
$$

and

$$
\frac{\left\langle U_{z}^{a b} \operatorname{tr}\left(t^{a} U_{x} t^{b} U_{y}^{\dagger}\right)\right\rangle}{2 N_{\mathrm{c}} C_{\mathrm{F}}}=e^{-\frac{N_{\mathrm{c}}}{2}\left(\mathcal{G}_{x z}+\mathcal{G}_{z y}-\mathcal{G}_{x y}\right)-C_{\mathrm{F}} \mathcal{G}_{x y}}
$$

where

$$
\mathcal{G}_{x y}(\eta)=(\mathcal{P}+i \mathcal{O})_{x y}
$$

whose real and imaginary parts are literally the $\mathcal{P}$ and $\mathcal{O}$ introduced as parametrization functions of the complex number $\left\langle\operatorname{tr}\left(U_{x} U_{y}^{\dagger}\right)\right\rangle(Y) / N_{\mathrm{c}}$. The truncation procedure asserts that they also consistently parametrize the $q \bar{q} g$ correlator and provides an explicit expression of $\mathcal{G}$ in terms of $G$ and $G^{d}$ and their respective initial conditions at $\eta_{0}$ :

$$
\begin{aligned}
\mathcal{P}_{x y}(\eta) & :=\int_{\eta_{0}}^{\eta} \mathrm{d} \eta^{\prime} G_{x y}+\mathcal{P}_{x y}\left(\eta_{0}\right)=\mathcal{P}_{y x}(\eta), \\
i \mathcal{O}_{x y}(\eta) & :=\frac{C_{\mathrm{d}}}{4} \int_{\eta_{0}}^{\eta} \mathrm{d} \eta^{\prime}\left(G_{y x x}^{d}-G_{y y x}^{d}\right)+i \mathcal{O}_{x y}\left(\eta_{0}\right) \\
& =-i \mathcal{O}_{y x}(\eta)
\end{aligned}
$$

Strikingly, the observables considered here do not allow access to $G_{u v w}$ with all three coordinates independent despite the fact that the $q \bar{q} g$ correlator features three distinct coordinates. For $G_{u v w}$ to occur with its full coordinate dependence one needs for example a protonlike state which remains outside the scope of this paper.

In the literature one often uses the same notation for the factorization rapidity $Y$ and the longitudinal coordinate in the path-ordered exponential $\eta$ (or $\ln x^{-}$). With the physical interpretation given above these are, however, not the same quantity. The coordinate $x^{-}$that gives the interpretation of the Wilson line as a path-ordered exponential in the color field is a spatial coordinate along the trajectory of the pathordered exponential. The local structure of the correlation function in the longitudinal coordinate essentially imposes the Gaussian property on the distribution of Wilson lines, since these are made up of independent infinitesimal increments. The coordinate $x^{-}$is, however, not directly 
related to an experimental observable, but cross sections always depend on Wilson lines integrated over $x^{-}$.

The rapidity $Y$, on the other hand, is a longitudinal momentum scale separating large- and small- $x$ degrees of freedom in the CGC formalism. The scale $Y$ is a factorization scale in the renormalization group evolution and should be chosen according to the typical longitudinal momentum scale in the studied scattering process. A loose uncertainty principle argument states that for a momentum space scale $Y$, the fields in the target are localized in a coordinate space interval $\Delta x^{-} \sim \exp \{Y\}$. Thus, identifying $\eta$ and $Y$ is indeed justified at the leading logarithmic level (but not at higher orders in perturbation theory). This identification is not needed or used anywhere in the present calculation and we will keep the separate notation for these two variables.

It is important to realize that Eq. (29) is a parametrization equation that expresses singlet Wilson line correlators in terms of a two-point function whose $Y$ dependence needs to be derived separately or is known a priori. Thus, the solutions of the parametrization equation, e.g. Eqs. (31a) and (31b), have an unknown dependence on the factorization rapidity $Y$ that will need to be derived from the actual QCD dynamics.

One way to derive an equation for the dependence of the two-point function $\mathcal{G}$ on the factorization rapidity $Y$ is to take the solutions of Eq. (29) and insert them into the appropriate equations from the Balitsky hierarchy. Every equation of the hierarchy leads to a different equation for $\mathcal{G}$. Choosing this equation to be the equation (21) for the expectation value of $\operatorname{tr}\left(U_{x} U_{y}^{\dagger}\right) / N_{c}$ dipole leads to a Gaussian truncation of the JIMWLK hierarchy.

It is also possible to rewrite the parametrization equation (30) in terms of a more abstract longitudinal coordinate, which can then be chosen to be equal to the evolution rapidity. This procedure can be used to simplify the coupled evolution equations for more complicated higher-point operators of Wilson lines [31], at the expense of losing the physical interpretation of the longitudinal coordinate. Here we will concentrate on the evolution equation for just the dipole operator and can easily remain with the physical interpretation of $\eta$ as being related to $x^{-}$ and distinct from the momentum rapidity $Y$.

As indicated in Eq. (32), the effective two-point functions do show the required symmetry properties of $\left\langle\operatorname{tr}\left(U_{x} U_{y}^{\dagger}\right)\right\rangle / N_{\mathrm{c}}$ under complex conjugation; one obtains a consistent gauge-invariant truncation of the associated Balitsky hierarchy with the evolution equation

$$
\frac{\mathrm{d}}{\mathrm{d} Y} \mathcal{G}_{x y}=\frac{\alpha_{\mathrm{s}}}{\pi^{2}} \int \mathrm{d}^{2} z \mathcal{K}_{x z y}\left(1-e^{-\frac{N_{\mathrm{c}}}{2}\left[\mathcal{G}_{x z}+\mathcal{G}_{z y}-\mathcal{G}_{x y}\right]}\right),
$$

which obviously couples real and imaginary parts. Equation (33) generalizes the large- $N_{\mathrm{c}}$ results of $[29,30]$ to finite $N_{\mathrm{c}}$.

The solutions to the parametrization equations (31) and thus the evolution equation (33) differ from their BK counterpart for the total cross section in the Gaussian truncation only through a nonvanishing imaginary part $i \mathcal{O} \neq 0$ appearing in $\mathcal{G}$. Indeed $i \mathcal{O}=0$ and $\mathcal{P} \in[0, \infty]$ is a consistent solution to this equation which leads to a successful phenomenology for HERA data at small $x$ [46]: If the initial condition for $\mathcal{G}$ is real, the equation never generates an imaginary part. This can be seen from the coupled equations for the real and imaginary parts explicitly:

$$
\begin{aligned}
& \frac{\mathrm{d}}{\mathrm{d} Y} \mathcal{P}_{x y}(Y)=\frac{\alpha_{\mathrm{s}}}{\pi^{2}} \int \mathrm{d}^{2} z K_{x z y}\left[1-e^{-\frac{N_{\mathrm{c}}}{2}\left[\mathcal{P}_{x z}+\mathcal{P}_{z y}-\mathcal{P}_{x y}\right](Y)} \cos \left(\frac{N_{\mathrm{c}}}{2}\left[\mathcal{O}_{x z}+\mathcal{O}_{z y}-\mathcal{O}_{x y}\right](Y)\right)\right], \\
& \frac{\mathrm{d}}{\mathrm{d} Y} \mathcal{O}_{x y}(Y)=\frac{\alpha_{\mathrm{s}}}{\pi^{2}} \int \mathrm{d}^{2} z K_{x z y}\left[e^{-\frac{N_{\mathrm{c}}}{2}\left[\mathcal{P}_{x z}+\mathcal{P}_{z y}-\mathcal{P}_{x y}\right](Y)} \sin \left(\frac{N_{\mathrm{c}}}{2}\left[\mathcal{O}_{x z}+\mathcal{O}_{z y}-\mathcal{O}_{x y}\right](Y)\right)\right] .
\end{aligned}
$$

\section{EVOLUTION IN THE THREE-POINT EXPONENTIAL TRUNCATION}

There are many different ways to rewrite the equation before implementing it numerically. The most prominent among these is the possibility to map (33) into the BK equation, as noted in [1]. Let us briefly recapitulate how this is done. Inserting the Gaussian correlator parametrizations of the three-point exponential truncation (31) into the first equation of the Balitsky hierarchy (21) and canceling an overall factor of $N_{\mathrm{c}}$, we get

$$
\begin{aligned}
\frac{\mathrm{d}}{\mathrm{d} Y} e^{-C_{\mathrm{F}} \mathcal{G}_{x y}}= & \frac{\alpha_{\mathrm{s}} C_{\mathrm{F}}}{\pi^{2}} \int \mathrm{d}^{2} z \tilde{\mathcal{K}}_{x z y} \\
& \times\left(e^{-\frac{N_{\mathrm{c}}}{2}\left[\mathcal{G}_{x z}+\mathcal{G}_{z y}-\mathcal{G}_{x y}\right]-C_{\mathrm{F}} \mathcal{G}_{x y}}-e^{-C_{\mathrm{F}} \mathcal{G}_{x y}}\right) .
\end{aligned}
$$

From here it is straightforward to arrive at Eq. (33) after canceling an overall factor $e^{-C_{\mathrm{F}} \mathcal{G}_{x y}}$ common to both sides.

To derive the relation between the BK equation and the Gaussian truncation one starts by multiplying both sides of Eq. (33) with $-\frac{N_{\mathrm{c}}}{2} e^{-\frac{N_{\mathrm{c}}}{2} \mathcal{G}_{x y}}$, leading to the alternative form 


$$
\begin{aligned}
\frac{\mathrm{d}}{\mathrm{d} Y} e^{-\frac{N_{\mathrm{c}}}{2} \mathcal{G}_{x y}}= & \frac{\alpha_{\mathrm{s}}}{\pi^{2}} \frac{N_{\mathrm{c}}}{2} \int \mathrm{d}^{2} z \mathcal{K}_{x z y} \\
& \times\left(e^{-\frac{N_{\mathrm{c}}}{2}\left[\mathcal{G}_{x z}+\mathcal{G}_{z y}\right]}-e^{-\frac{N_{\mathrm{c}}}{2} \mathcal{G}_{x y}}\right)
\end{aligned}
$$

for this evolution equation. Now, after identifying

$$
S_{x y}^{\mathrm{BK}}:=e^{-\frac{N_{\mathrm{c}}}{2} \mathcal{G}_{x y}},
$$

it is manifest that Eq. (36) is equivalent to the BK equation

$$
\frac{\mathrm{d}}{\mathrm{d} Y} S_{x y}^{\mathrm{BK}}=\frac{\alpha_{\mathrm{s}}}{\pi^{2}} \frac{N_{\mathrm{c}}}{2} \int \mathrm{d}^{2} z \tilde{\mathcal{K}}_{x z y}\left(S_{x z}^{\mathrm{BK}} S_{z y}^{\mathrm{BK}}-S_{x y}^{\mathrm{BK}}\right) .
$$

Equations (33), (35), (36), and (38) are all equivalent: They all determine the evolution for the same function $\mathcal{G}$ and will lead to the same $Y$ dependence provided we set the same initial condition on $\mathcal{G}$. The difference between the Gaussian truncation and the large- $N_{\mathrm{c}}$ BK equation (in the sense the term is commonly used) is in the relation between the physical scattering amplitude and the solution of the evolution equation. In the large- $N_{\mathrm{c}}$ limit the solution of the BK equation $S^{\mathrm{BK}}$ is assumed to be the physical scattering amplitude. In the finite- $N_{\mathrm{c}}$ Gaussian truncation the physical scattering amplitude is related to the fundamental two-point correlator $\mathcal{G}$ by (33) and to the solution of the BK equation by (36). Thus the physical scattering amplitude is obtained from the solution of the BK equation as

$$
S_{x y}=\left\langle\frac{\operatorname{tr}\left(U_{x} U_{y}^{\dagger}\right)}{N_{\mathrm{c}}}\right\rangle=\left(S_{x y}^{\mathrm{BK}}\right)^{\frac{2 C_{\mathrm{F}}}{N_{\mathrm{c}}}}
$$

Thus the real and imaginary parts of the physical dipole operator in the Gaussian truncation $S_{x y}$, expressed in terms of the real and imaginary parts of the BK-evolved dipole, read

$$
\begin{aligned}
& \operatorname{Re} S_{x y}=\left|S_{x y}^{\mathrm{BK}}\right|^{\frac{2 C_{\mathrm{F}}}{N_{\mathrm{c}}}} \cos \left\{\frac{2 C_{\mathrm{F}}}{N_{\mathrm{c}}} \arctan \frac{\operatorname{Im} S_{x y}^{\mathrm{BK}}}{\operatorname{Re} S_{x y}^{\mathrm{BK}}}\right\}, \\
& \operatorname{Im} S_{x y}=\left|S_{x y}^{\mathrm{BK}}\right|^{\frac{2 C_{\mathrm{F}}}{N_{\mathrm{c}}}} \sin \left\{\frac{2 C_{\mathrm{F}}}{N_{\mathrm{c}}} \arctan \frac{\operatorname{Im} S_{x y}^{\mathrm{BK}}}{\operatorname{Re} S_{x y}^{\mathrm{BK}}}\right\} .
\end{aligned}
$$

Note that a purely real solution of the BK equation still gives a purely real dipole expectation value, but the presence of an imaginary part in the BK equation affects both the real and imaginary parts of the physical dipole.

Separating the identity from the BK-equation dipole operator $S^{\mathrm{BK}}$, one gets the scattering amplitude $N_{r}^{\mathrm{BK}}=$ $1-S_{r}^{\mathrm{BK}}$, where $\boldsymbol{r}=\boldsymbol{x}-\boldsymbol{y}$. In line with our earlier conventions in Eq. (9) we denote its real and imaginary parts as $P_{r}^{\mathrm{BK}}:=\operatorname{Re}\left(N_{r}^{\mathrm{BK}}\right)$, the BK Pomeron and $O_{r}^{\mathrm{BK}}:=\operatorname{Im}\left(N_{r}^{\mathrm{BK}}\right)$, the BK odderon. In terms of these, the evolution equation (38) now becomes a set of two real integro-differential equations

$$
\begin{aligned}
\frac{\mathrm{d} P_{\boldsymbol{r}}^{\mathrm{BK}}}{\mathrm{d} Y}=\frac{\alpha_{\mathrm{s}} N_{\mathrm{c}}}{2 \pi^{2}} \int \mathrm{d}^{2} \boldsymbol{r}^{\prime} \frac{\boldsymbol{r}^{2}}{\boldsymbol{r}^{\prime 2} \boldsymbol{r}^{\prime \prime 2}}\left(P_{\boldsymbol{r}^{\prime}}^{\mathrm{BK}}+P_{\boldsymbol{r}^{\prime \prime}}^{\mathrm{BK}}\right. \\
\left.-P_{\boldsymbol{r}}^{\mathrm{BK}}-P_{\boldsymbol{r}^{\prime}}^{\mathrm{BK}} P_{\boldsymbol{r}^{\prime \prime}}^{\mathrm{BK}}+O_{\boldsymbol{r}^{\prime}}^{\mathrm{BK}} O_{\boldsymbol{r}^{\prime \prime}}^{\mathrm{BK}}\right), \\
\frac{\mathrm{d} O_{\boldsymbol{r}}^{\mathrm{BK}}}{\mathrm{d} Y}=\frac{\alpha_{\mathrm{s}} N_{\mathrm{c}}}{2 \pi^{2}} \int \mathrm{d}^{2} \boldsymbol{r}^{\prime} \frac{\boldsymbol{r}^{2}}{\boldsymbol{r}^{\prime 2} \boldsymbol{r}^{\prime 2}}\left(O_{\boldsymbol{r}^{\prime}}^{\mathrm{BK}}+O_{\boldsymbol{r}^{\prime \prime}}^{\mathrm{BK}}-O_{\boldsymbol{r}}^{\mathrm{BK}}\right. \\
\left.-P_{\boldsymbol{r}^{\prime}}^{\mathrm{BK}} O_{\boldsymbol{r}^{\prime \prime}}^{\mathrm{BK}}-O_{\boldsymbol{r}^{\prime}}^{\mathrm{BK}} P_{\boldsymbol{r}^{\prime \prime}}^{\mathrm{BK}}\right),
\end{aligned}
$$

where $\boldsymbol{r}^{\prime \prime} \equiv \boldsymbol{r}-\boldsymbol{r}^{\prime}$. These equations are identical to those derived in Refs. $[29,30]$ in the large- $N_{\mathrm{c}}$ limit. As discussed above, what changes at finite $N_{\mathrm{c}}$ in the Gaussian truncation is the relation between the solution of these equations and the physical scattering amplitude, which is now given by Eqs. (40) and (41).

Recall that from the definition of $S_{r}$ it follows that $S_{r}^{*}=S_{-r}$. This in turn imposes separate symmetry properties on the real and imaginary parts of $\mathcal{G}=\mathcal{P}+i \mathcal{O}$ which are correctly reproduced by our truncation in Eq. (32). Via (37) they directly imply that $S_{r}^{\mathrm{BK} *}=S_{-r}^{\mathrm{BK}}$. Thus the real and imaginary parts of the amplitude are odd or even under reflections:

$$
\begin{gathered}
P_{-r}^{\mathrm{BK}}=P_{r}^{\mathrm{BK}}, \\
O_{-r}^{\mathrm{BK}}=-O_{r}^{\mathrm{BK}} .
\end{gathered}
$$

For the linear BFKL part of the equation it is particularly convenient to decompose the solution to the evolution equation in terms of eigenfunctions of the kernel, both in $|\boldsymbol{r}|$ and in azimuthal angle. For the nonlinear case it is more convenient to continue working in $r$ space, but we can still perform a Fourier series expansion in the azimuthal angle. The symmetry (44) and (45) dictates that the Pomeron and odderon can only have even or odd harmonics, respectively:

$$
\begin{gathered}
P_{r}^{\mathrm{BK}}=\sum_{n=0}^{\infty} P_{2 n}^{\mathrm{BK}}(r) \cos \left(2 n \varphi_{\boldsymbol{r}}\right), \\
O_{r}^{\mathrm{BK}}=\sum_{n=0}^{\infty} O_{2 n+1}^{\mathrm{BK}}(r) \cos \left((2 n+1) \varphi_{\boldsymbol{r}}\right),
\end{gathered}
$$

where $\varphi_{r}$ is the angle of the vector $\boldsymbol{r}$ with respect to an (arbitrary) reaction plane. Based on the known BFKL dynamics in the linear regime, we expect the small azimuthal harmonics to dominate in the high energy regime. Our working hypothesis here is that the same is true also in the nonlinear case. This allows us to efficiently study the equations by truncating the series and only keeping the lowest harmonics. We can control the error made in this approximation ex post by calculating the rapidity dependence of the first neglected term in the series from the ones that are kept. 
A quick examination of Eqs. (42) and (43) reveals that the usual azimuthal $P_{0}^{\mathrm{BK}}(r)$ is a fixed point of the equation. Including the lowest odderon harmonic $O_{1}^{\mathrm{BK}}(r)$ will generate a second Pomeron $P_{2}^{\mathrm{BK}}(r)$ through the $\left(O^{\mathrm{BK}}(r)\right)^{2}$ term in (42), which again will generate a higher $O_{3}^{\mathrm{BK}}(r)$ harmonic through the nonlinear $P^{\mathrm{BK}} O^{\mathrm{BK}}$ coupling in (43). In this way, including an odderon will automatically generate an infinite tower of higher harmonics in both the odderon and the Pomeron amplitudes. In principle, it could thus be possible that, through this coupling, an odderon component would have an observable signal in a $P$-even observable such as dijet correlations at an electron-ion collider [47].

For this first numerical study we have truncated the series to the smallest harmonics of both the Pomeron and odderon, i.e. $P_{0}^{\mathrm{BK}}(r)$ and $O_{1}^{\mathrm{BK}}(r)$, by dropping the $O_{r^{\prime}}^{\mathrm{BK}} O_{r^{\prime \prime}}^{\mathrm{BK}}$ term from the Pomeron evolution equation (42). With this truncation, the Pomeron amplitude stays rotationally invariant and the odderon equation (43) can be solved as such with only the lowest harmonic $O_{1}^{\mathrm{BK}}(r)$. To see this explicitly, note that with only the azimuthally symmetric component in the Pomeron amplitude, we can write the odderon equation as

$$
\begin{aligned}
\frac{\mathrm{d} O_{1}^{\mathrm{BK}}(r) \cos \theta}{\mathrm{d} Y}= & \frac{\alpha_{\mathrm{s}} N_{\mathrm{c}}}{2 \pi^{2}} \int \mathrm{d}^{2} \boldsymbol{r}^{\prime} \frac{\boldsymbol{r}^{2}}{\boldsymbol{r}^{\prime 2} \boldsymbol{r}^{\prime \prime 2}} \times\left(O_{1}^{\mathrm{BK}}\left(r^{\prime}\right) \cos \theta^{\prime}+O_{1}^{\mathrm{BK}}\left(r^{\prime \prime}\right) \cos \theta^{\prime \prime}-O_{1}^{\mathrm{BK}}(r) \cos \theta\right. \\
& \left.-P_{0}^{\mathrm{BK}}\left(r^{\prime}\right) O_{1}^{\mathrm{BK}}\left(r^{\prime \prime}\right) \cos \theta^{\prime \prime}-O_{1}^{\mathrm{BK}}\left(r^{\prime}\right) P_{0}^{\mathrm{BK}}\left(r^{\prime \prime}\right) \cos \theta^{\prime}\right),
\end{aligned}
$$

where $\theta, \theta^{\prime}$ and $\theta^{\prime \prime}$ are the angles of $\boldsymbol{r}, \boldsymbol{r}^{\prime}$ and $\boldsymbol{r}^{\prime \prime}$ with respect to the $x$ axis. In practice we can solve Eq. (43) by choosing the vector $r$ to lie on the $x$ axis, with thus $\cos \varphi_{r}=1$. To show explicitly that this is the case, we use $\left(r^{\prime \prime}\right)^{2}=r^{2}+\left(r^{\prime}\right)^{2}-2 r r^{\prime} \cos \left(\theta-\theta^{\prime}\right)$ and $\cos \theta^{\prime \prime}=\left(r \cos \theta-r^{\prime} \cos \theta^{\prime}\right) / r^{\prime \prime}$ to write this as

$$
\begin{aligned}
\frac{\mathrm{d} O_{1}^{\mathrm{BK}}(r) \cos \theta}{\mathrm{d} Y}= & \frac{\alpha_{\mathrm{s}} N_{\mathrm{c}}}{2 \pi^{2}} \int \mathrm{d} r^{\prime} \mathrm{d} \theta^{\prime} r^{\prime} \frac{r^{2}}{\left.\left(r^{\prime}\right)^{2}\left(r^{\prime \prime}\right)^{2}\right)} \times\left[\left(1-P_{0}^{\mathrm{BK}}\left(r^{\prime \prime}\right)\right) O^{\mathrm{BK}}\left(r^{\prime}\right) \cos \theta^{\prime}\right. \\
& \left.+\left(1-P_{0}^{\mathrm{BK}}\left(r^{\prime}\right)\right) O_{1}^{\mathrm{BK}}\left(r^{\prime \prime}\right) \frac{r \cos \theta-r^{\prime} \cos \theta^{\prime}}{r^{\prime \prime}}-O_{1}^{\mathrm{BK}}(r) \cos \theta\right] .
\end{aligned}
$$

We then take $\phi=\theta^{\prime}-\theta$ as a new integration variable and use the identity $\cos \left(\theta^{\prime}\right)=\cos \phi \cos \theta-\sin \phi \sin \theta$. Now the terms that are proportional to $\sin \theta$ are also proportional to $\sin \phi$ times an even function of $\phi$ and vanish upon integration over $\phi$, leaving every term on the right-hand side of Eq. (49) proportional to $\cos \theta$. Thus, as discussed earlier, with this approximation of a $\theta$-independent Pomeron amplitude the equation for the $\cos \theta$ harmonic of the odderon closes. We can cancel the $\cos \theta$ from Eq. (49), and we are left with the truncated set of equations

$$
\begin{aligned}
& \frac{\mathrm{d} P_{0}^{\mathrm{BK}}(r)}{\mathrm{d} Y}=\frac{\alpha_{\mathrm{s}} N_{\mathrm{c}}}{2 \pi^{2}} \int \mathrm{d}^{2} \boldsymbol{r}^{\prime} \frac{\boldsymbol{r}^{2}}{\boldsymbol{r}^{\prime 2} \boldsymbol{r}^{\prime 2}}\left(P_{0}^{\mathrm{BK}}\left(r^{\prime}\right)+P_{0}^{\mathrm{BK}}\left(r^{\prime \prime}\right)-P_{0}^{\mathrm{BK}}(r)-P_{0}^{\mathrm{BK}}\left(r^{\prime}\right) P_{0}^{\mathrm{BK}}\left(r^{\prime \prime}\right)\right), \\
& \frac{\mathrm{d} O_{1}^{\mathrm{BK}}(r)}{\mathrm{d} Y}=\frac{\alpha_{\mathrm{s}} N_{\mathrm{c}}}{2 \pi^{2}} \int \mathrm{d} r^{\prime} \mathrm{d} \phi r^{\prime} \frac{r^{2}}{\left.\left(r^{\prime}\right)^{2}\left(r^{\prime \prime}\right)^{2}\right)}\left[\left(1-P_{0}^{\mathrm{BK}}\left(r^{\prime \prime}\right)\right) O^{\mathrm{BK}}\left(r^{\prime}\right) \cos \phi+\left(1-P_{0}^{\mathrm{BK}}\left(r^{\prime}\right)\right) O_{1}^{\mathrm{BK}}\left(r^{\prime \prime}\right) \frac{r-r^{\prime} \cos \phi}{r^{\prime \prime}}-O_{1}^{\mathrm{BK}}(r)\right],
\end{aligned}
$$

with $\left(r^{\prime \prime}\right)^{2}=r^{2}+\left(r^{\prime}\right)^{2}-2 r r^{\prime} \cos \phi$.

We will now proceed to numerically solve Eqs. (50) and (51). We will parametrize the initial condition as in Eq. (19):

$$
\begin{gathered}
\left.P_{0}^{\mathrm{BK}}\left(r^{\prime \prime}\right)\right|_{Y=0}=1-\exp \left\{\frac{-Q_{0}^{2} r^{2}}{4}\right\}, \\
\left.O_{1}^{\mathrm{BK}}(r)\right|_{Y=0}=-\kappa \exp \left\{\frac{-Q_{0}^{2} r^{2}}{4}\right\}\left(\frac{Q_{0}^{3} r^{3}}{8}\right),
\end{gathered}
$$

with the maximal value $\kappa=1 / 3$. Figure 8 shows the resulting amplitudes. For the Pomeron part one sees the familiar "traveling wave" solution moving towards smaller dipoles with rapidity. The odderon amplitude, on the other hand, merely decreases in magnitude but its characteristic dipole size scale does not decrease. This behavior is quantified further in Figs. 9 and 10, showing the height of the odderon amplitude peak as a function of rapidity and the ratio of the (BK) odderon to the (BK) Pomeron amplitude.

In the calculations presented above, we have neglected the odderon squared term in the evolution equation. This can be justified by the fact that, since it has not yet been unambiguously observed experimentally, the odderon amplitude can be expected to be small. Also expectations based on the linear evolution equation would lead to an odderon amplitude that 

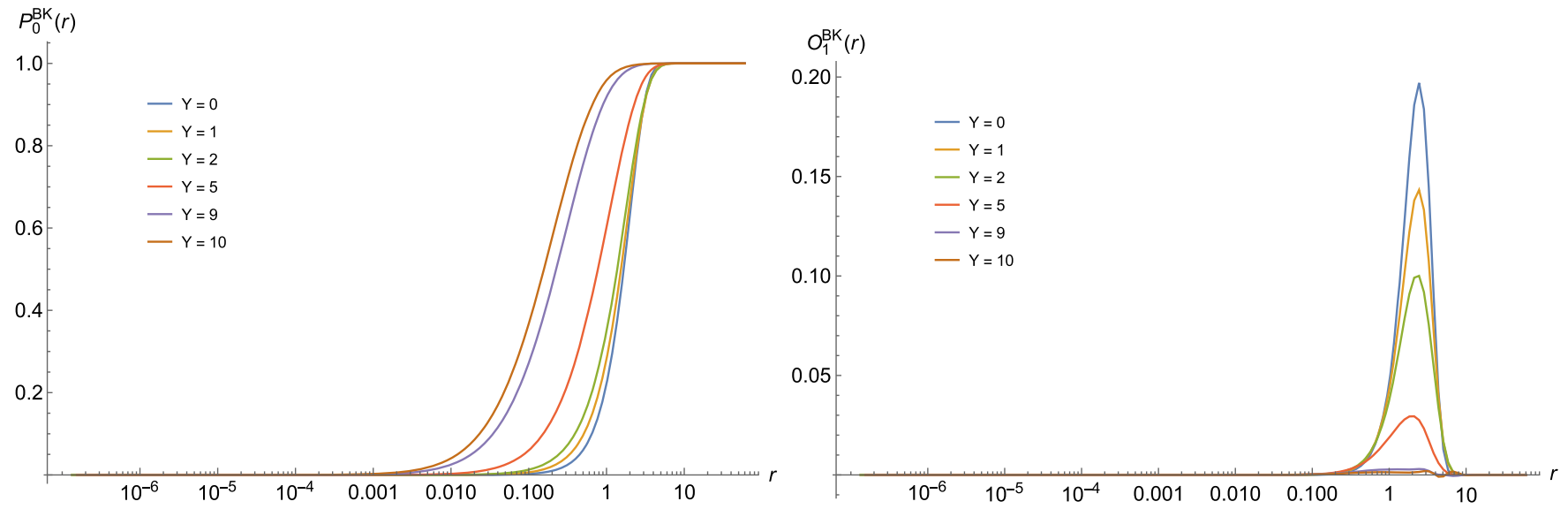

FIG. 8. Evolution of the Pomeron (left) and odderon (right) amplitudes according to Eqs. (50) and (51) with the initial condition (19) with $\kappa=1 / 3$.

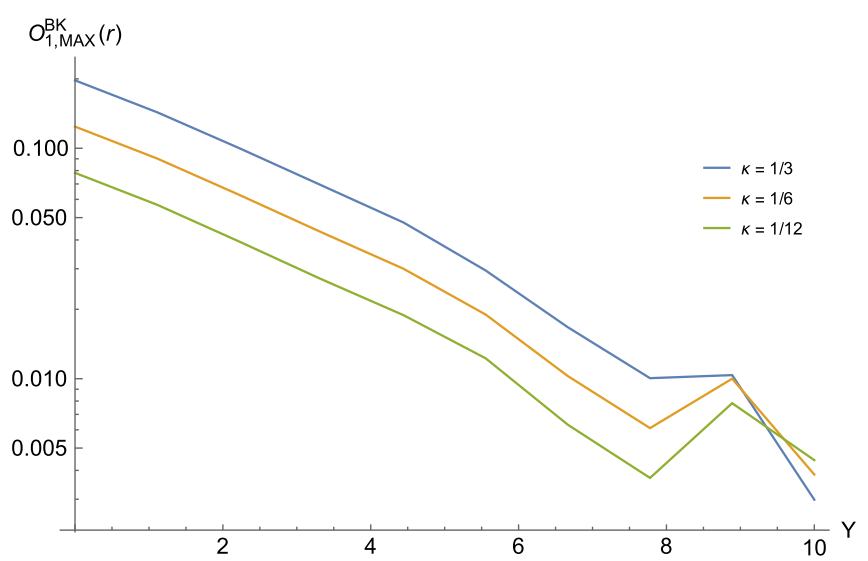

FIG. 9. Plot of the height of the odderon peak (as shown in Fig. 8) as a function of rapidity. Three different values $\kappa=1 / 3$, $1 / 6$ and $1 / 12$ are shown.

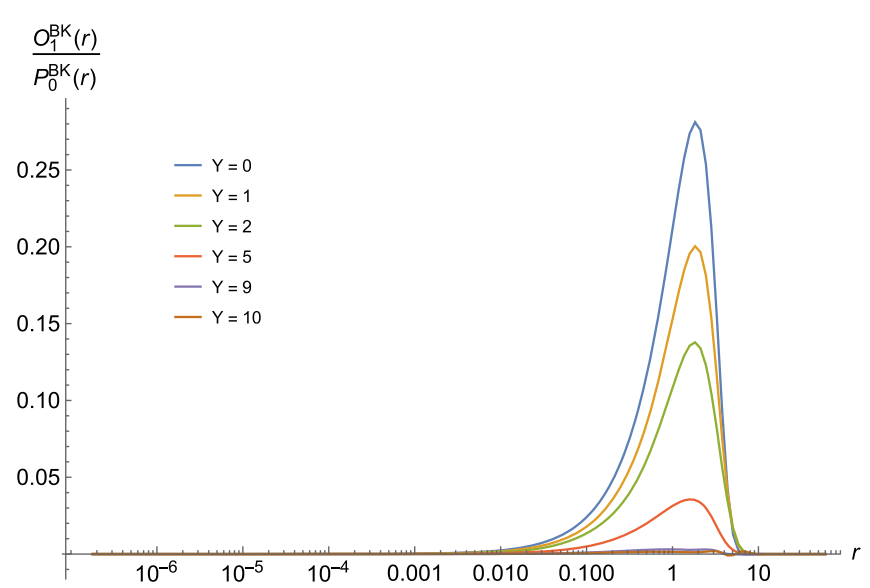

FIG. 10. Ratio of $\mathcal{O}(r) / \mathcal{P}(r)$ for $\kappa=1 / 3$ as a function of $r$ at different rapidities. decreases as a function of rapidity [29,30]. Within the truncation of the harmonic series (46) and (47) one can estimate the size of this approximation by evaluating the contribution of the first neglected term on one time step, i.e. the contribution of the odderon squared term to the evolution equation of the Pomeron. As discussed previously, the square of the odderon term $O_{1}^{\mathrm{BK}}(r) \cos \theta$ gives both a $\theta$-independent and a $\cos 2 \theta$ contribution to the evolution equation of the Pomeron. We denote the coefficients of these by $a_{1}$ and $a_{2}$; i.e. we write

$$
\frac{\mathrm{d} P_{r}^{\mathrm{BK}}}{\mathrm{d} Y}=[\mathrm{BK}]+a_{1}(r)+a_{2}(r) \cos \left(2 \theta_{r}\right)
$$

We can now compare the odderon terms to the rotationally invariant solution. Figure 11 shows the initial condition for the fairly large value of $\kappa=1 / 3$. It can be seen that the odderon squared terms are negligible in the small- $r$ region that drives the evolution. The $\theta$-independent $a_{1}$ term is particularly small, while $a_{2}$ is slightly larger. We conclude

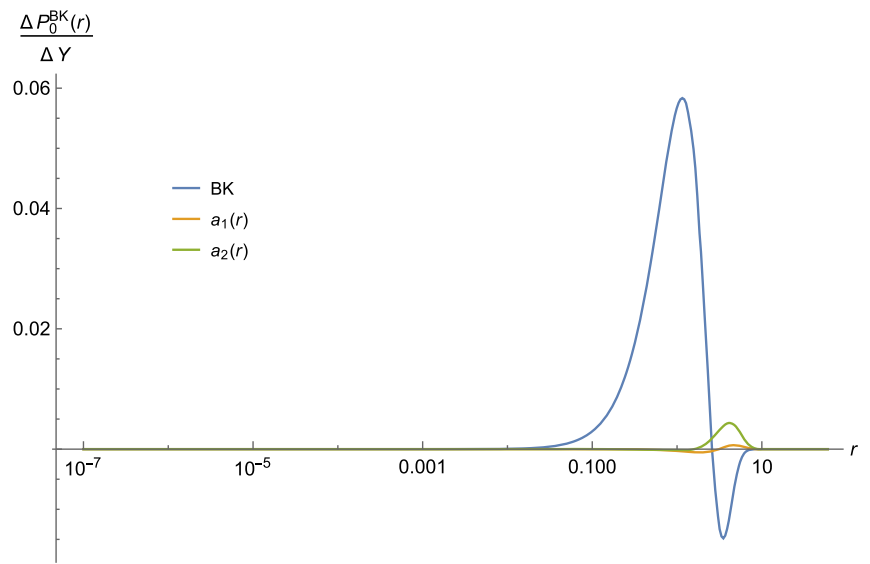

FIG. 11. Contribution to $\Delta \mathcal{P}_{r} / \Delta Y$ in one step in rapidity from: the BK equation, $a_{1}(r)$ and $a_{2}(r)$, with the maximal odderon amplitude $\kappa=1 / 3$. 
that the main effect of the nonlinear odderon term is not to modify the evolution of the rotationally invariant $P_{0}^{\mathrm{BK}}(r)$ amplitude but to introduce a small $\cos 2 \theta$ term into the Pomeron.

\section{FULL LEADING-ORDER JIMWLK EVOLUTION WITH ODDERON ADMIXTURE}

Since the evolution equation (33) or (34) is the result of a truncation of the full functional JIMWLK evolution, one may legitimately ask if the truncation deviates quantitatively or even qualitatively from a full JIMWLK simulation. This can be performed at leading order or at partial next-to-leading order with running coupling corrections included. Since in this paper we are only interested in qualitative behavior we have, for simplicity, chosen the former option.

What is quite remarkable is that the only thing that needs to change to perform a simulation run is the initial condition. The Langevin simulation governing JIMWLK evolution is carried out on a square transverse grid. A simulation for the total cross section starts from an initial condition that treats both of the principal directions of the transverse plane in the same way. To obtain an odderon admixture one must break this lattice remnant of rotational invariance and introduce a bias towards one of the directions into the initial condition - the code used for evolution needs no modification at all.

In this work we investigate the properties of the parityodd initial states with the following simple setup: We use a lattice of size $L^{2}$ with periodic boundary conditions. First we generate an ensemble of standard parity-even initial states with a probability distribution

$$
P\left(\operatorname{Retr}\left(U_{\boldsymbol{x}} U_{\boldsymbol{y}}^{\dagger}\right)\right) \propto \exp \left(-(x-y)^{2} / 4 R^{2}\right),
$$

using the methods described in [19]. This generates configurations with saturation scale $Q_{\mathrm{s}} \sim 1 / R$. The expectation value of the imaginary part of $\left\langle\operatorname{tr}\left(U_{x} U_{y}^{\dagger}\right)\right\rangle / N_{\mathrm{c}}$ vanishes and its real part falls into the interval between 0 and 1 . Such an ensemble is a suitable starting point for a simulation without an odderon admixture.

To introduce an odderon contribution one needs to generate an imaginary part. A convenient way of doing so is to consider a "potential"

$$
V[U](\boldsymbol{x})=\alpha \sum_{y} \operatorname{Imtr}\left(U_{x} U_{\boldsymbol{y}}^{\dagger}\right) f(\boldsymbol{x}-\boldsymbol{y}),
$$

where $\alpha$ is a small real parameter and $f$ is an odd function of $\boldsymbol{r}$ that breaks the symmetry between the two coordinate directions:

$$
f(\boldsymbol{x})=x_{1} e^{-x^{2} / 4 R^{2}} \frac{\left(x_{1}^{2}-(L / 2)^{2}\right)\left(x_{2}^{2}-(L / 2)^{2}\right)}{L^{4}} .
$$

The last part of the expression merely ensures that $f(x)$ vanishes at the boundaries $x_{i}= \pm L / 2$ so that no discontinuities arise there.

We use a left derivative to define the force induced by the "potential" $V$ as

$$
F_{a}(x)=-\alpha \sum_{y} \operatorname{Retr}\left(\lambda_{a} U_{x} U_{y}^{\dagger}\right) f(x-y)
$$

and make an update $U_{\boldsymbol{x}} \rightarrow e^{i \alpha F_{a}(x) \lambda_{a}} U_{\boldsymbol{x}}$. This update is repeated a few times for all sites $\boldsymbol{x}$. The magnitude of the parity-odd contribution can be modified by adjusting the constant $\alpha$ and the number of update steps.

In Figs. 12-14 we show the behavior of the real and imaginary parts of $\operatorname{tr}\left(U_{\boldsymbol{x}} U_{\boldsymbol{y}}^{\dagger}\right)$ as measured on $L^{2}=128^{2}$ lattices, using (a) no, (b) mild and (c) very large odderon contributions, respectively. Let us discuss the three cases in turn:
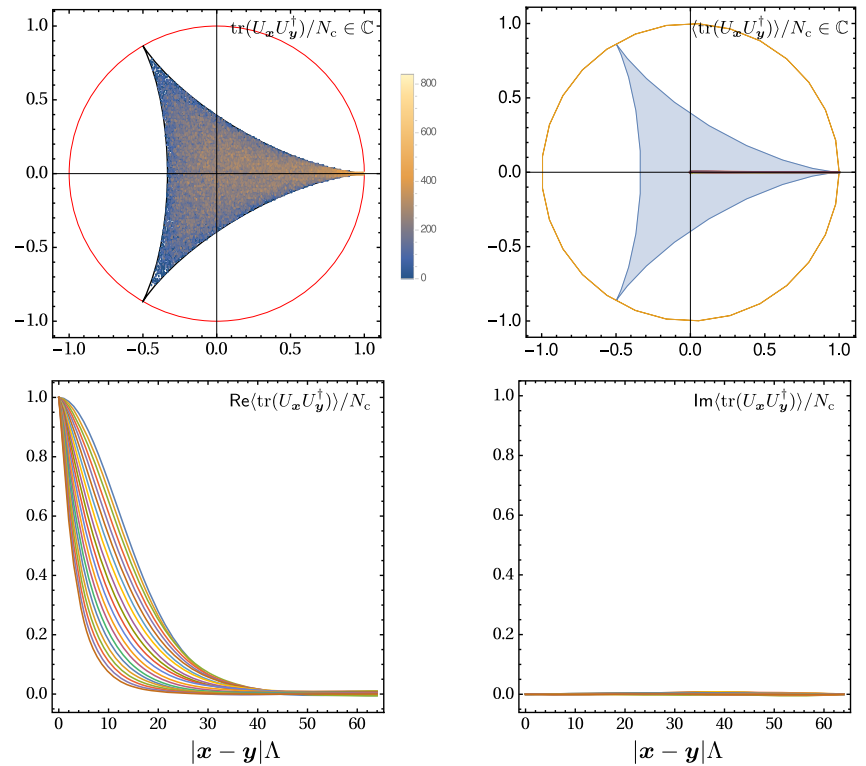

FIG. 12. Leading-order Langevin simulations with curves for different $Y$ values at $N_{\mathrm{c}}=3$ without an odderon admixture. The top row shows a density histogram of individual trace values (left) and averages (right) for configurations pulled from the ensembles through some $Y$ range. The dipole $\operatorname{tr}\left(U_{x} U_{y}^{\dagger}\right) / N_{\mathrm{c}}$ becomes 1 in the short-distance limit. This is why the distribution exhibits a strong maximum there. Note that the configurations cover much of the allowed range with only a small fraction falling near the maximally anticorrelated corners at $e^{ \pm i 2 \pi / 3}$. The density distribution is symmetric under reflection about the real axis. The averages fall into the real interval $[0,1]$, despite the fact that many individual configurations show negative real parts. The bottom row shows real and imaginary parts of the correlator averages for a number of $Y$ values. The real part exhibits the familiar approach to scaling (curves move "left" with increasing $Y$ ) and an imaginary part that is zero within good accuracy with only small fluctuations visible. 

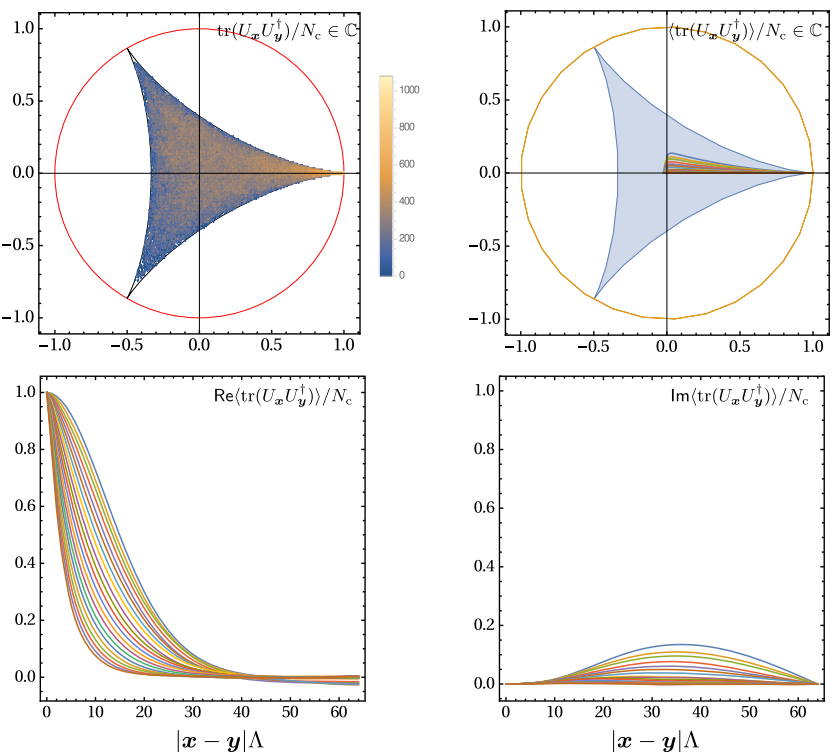

FIG. 13. Leading-order Langevin simulations with curves for different $Y$ values at $N_{\mathrm{c}}=3$ with a moderate odderon admixture. The top row shows a density histogram of individual trace values (left) and averages (right) for configurations pulled from the ensembles through some $Y$ range. Note that, as in Fig. 12, the configurations cover much of the allowed range with only a small fraction falling near the maximally anticorrelated corners at $e^{ \pm i 2 \pi / 3}$. The density distribution now shows a small bias towards positive real parts that leads to a nontrivial imaginary part in the averages. These move towards the real axis as $Y$ increases. The bottom row shows real and imaginary parts of the correlator averages for a number of $Y$ values. The real part now shows a small amount of anticorrelation which is erased quickly. The overall trend is an approach to scaling behavior very similar to that of the odderon-free simulation of Fig. 12. The imaginary part is small and erased in place as $Y$ increases. The two contributions behave in a qualitatively different manner: approach to scaling for the real part (the Pomeron) decay for the imaginary part (the odderon).

(a) No odderon in the initial state.-The ensemble is generated to follow Eq. (42) with no distortion applied so that the average

$$
\left\langle\operatorname{tr}\left(U_{x} U_{y}^{\dagger}\right)\right\rangle(Y) / N_{\mathrm{c}} \in[0,1] \text { for all } Y .
$$

The imaginary part vanishes in the initial condition and none is generated during evolution. This can likely be traced back to the adjoint nature of the Wilson lines "dressing" the Gaussian noise in the Langevin version of the leading-order JIMWLK equation. Note that this holds despite the fact that individual configurations $\operatorname{tr}\left(U_{x} U_{y}^{\dagger}\right) / N_{\mathrm{c}}$ occur anywhere inside the hypocycloid allowed by the constraints discussed in Sec. III. Plots illustrating this simulation are shown in Fig. 12. The top left displays a density histogram of configurations $\operatorname{tr}\left(U_{x} U_{y}^{\dagger}\right) / N_{\mathrm{c}}$ which almost fill the whole allowed
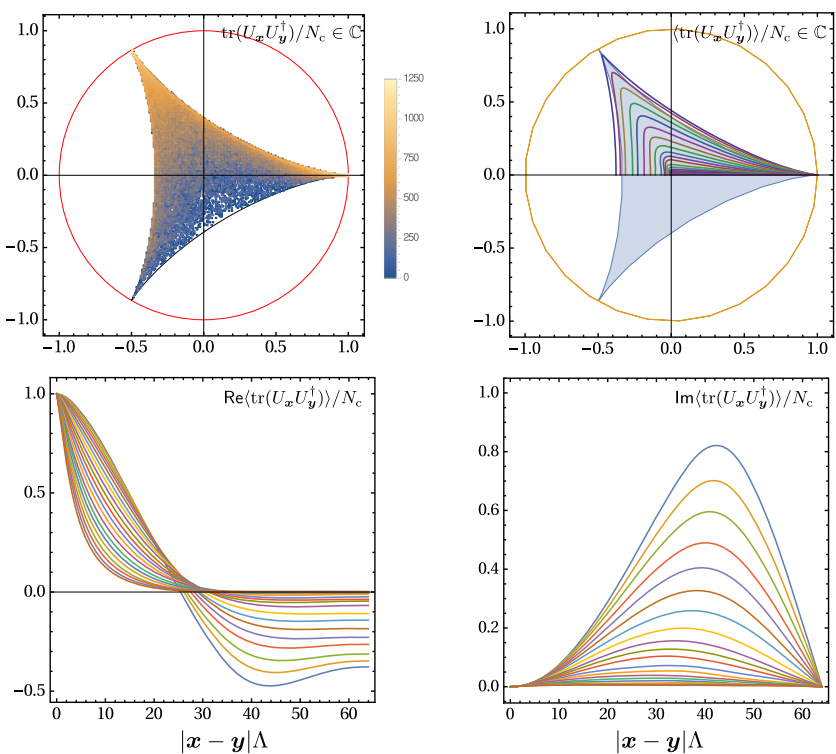

FIG. 14. Leading-order Langevin simulations with curves for different $Y$ values at $N_{\mathrm{c}}=3$ with a maximized odderon admixture. The layout repeats that of Figs. 12 and 13. The density histogram now shows a second maximum at $e^{i 2 \pi / 3}$, a strong anticorrelating distortion of the initial condition. This manifests itself in averages that (initially) push outside the hypocycloid into the triangle connecting the 3rd roots of unity, as discussed in the text. With this come strong anticorrelations in the real parts in the initial condition. These features that are extreme in the initial conditions nevertheless are erased during evolution which again approaches scaling form. The imaginary parts are maximized in the initial condition beyond what we expect to be physical, but the overall behavior is the same as for a moderate odderon admixture: The odderon contribution is erased in place.

region. Note that the density is lowest at the anticorrelated cusps corresponding to $e^{ \pm i 2 \pi / 3} \mathbb{1}$ and that the distribution is symmetric under a reflection along the real axis. As a consequence, Eq. (59) is satisfied for all $Y$, as shown top right. The plots in the second row show real and imaginary parts as a function of dipole size. $^{3}$ The real part shows the familiar decay of the correlation length $R_{\mathrm{S}}(Y)$ and will develop scaling behavior if allowed to evolve far enough.

(b) A moderate odderon admixture to the initial state.Plots illustrating this simulation are shown in Fig. 13. The density histogram for individual configurations $\operatorname{tr}\left(U_{x} U_{y}^{\dagger}\right) / N_{\mathrm{c}}$ shows a bias towards a positive imaginary part, which is confirmed by the averages $\left\langle\operatorname{tr}\left(U_{x} U_{y}^{\dagger}\right)\right\rangle(Y) / N_{\mathrm{c}}$ shown top right. Along with the appearance of an imaginary part, one observes that the real part shows negative values-anticorrelations appear. This simulation is qualitatively close to what we have discussed in the context of the truncations

${ }^{3}$ Units can only be assigned after a data fit, which is not the goal of this publication. 
with a perturbative boundary condition, although no effort was made to create a literal $r^{3}$ behavior for the initial condition.

The striking feature of this is the qualitatively different behavior seen in the $Y$ dependence of real and imaginary parts. While the real part does exhibit a small anticorrelation for $r \gtrsim R_{\mathrm{S}} \equiv 1 / Q_{\mathrm{s}}$, the evolution of $R_{\mathrm{S}}$ and the approach to scaling are affected very little by the presence of an imaginary part. The imaginary part is not characterized by a moving $Y$ dependent scale; it shows a single, clearly developed maximum that remains largely at the same distance scale as $Y$ changes. The dominating feature of evolution is that the height of the maximum shrinks - the odderon is erased in place.

(c) A maximized odderon admixture in the initial state.Plots illustrating this simulation are shown in Fig. 14. The initial condition is maximally distorted and the distribution of trace values has developed a second maximum in the maximally anticorrelated region at $e^{i 2 \pi / 3} \mathbb{1}$.

This is an extreme case that we have included to illustrate the features of JIMWLK evolution. Due to the extreme initial condition, the dipole averages start to fall outside the hypocycloids and the lattice is too small for the real part to reach zero at large distances. Nevertheless, the behavior of the imaginary part still mirrors that of the realistic odderon admixture: The odderon does not move; it decays in place. We conclude that both the scale shift for the real part and the fixed scale decay for the imaginary part of the dipole correlator are genuine features of JIMWLK evolution, irrespective of the details of the initial condition.

\section{DISCUSSION}

In this paper we have shown how to derive the high energy evolution equations for the odderon amplitude using a consistent three-point truncation of the Balitsky hierarchy. In the large- $N_{\mathrm{c}}$ limit our solution recovers that of $[29,30]$. Decomposing the amplitudes in terms of Fourier harmonics yields an infinite series of coupled equations. Due to the nonlinear relation between the real and imaginary parts of the physical scattering amplitude, and the solutions of the corresponding BK equation [see Eqs. (40) and (41)], the presence of any odderon component introduces an angular dependence at all harmonics $n$ into the scattering amplitude. This correlation vanishes in the large- $N_{\mathrm{c}}$ limit and would therefore not have been accessible previously. This coupling between the odderon amplitude and higher harmonics could allow for a quantitative experimental access to the odderon component in multiparticle correlations in future precise DIS experiments [48].

By truncating the harmonic series to the first nontrivial terms for both the Pomeron and odderon parts, one gets a closed nonlinear equation for the energy dependence of the odderon amplitude. We have presented the first numerical solution to this equation available in the literature. We have then completely independently confirmed these results with a numerical lattice solution of the full JIMWLK equation with an initial condition containing an odderon component. Both of these numerical calculations have confirmed the earlier analytical conjectures based on the linear BFKL limit [29,30], showing that the odderon amplitude decreases with increasing collision energy. This observation justifies the truncation of the higher harmonic terms used in the BK-like numerical evaluation (the JIMWLK simulation needs no such truncation).

The odderon appears in full JIMWLK evolution only by preparing its initial conditions in a way that breaks rotational invariance in the transverse plane. This mirrors directly what is happening in a measurement process: The ensemble we average over needs to break rotation invariance in both cases. In an experiment this can be achieved by measuring polarizations, as is done in an STSA measurement. The total cross section as an average over a fully symmetric unbiased set of events will not be able to couple to the odderon at all. (Not even through mixing of real and imaginary parts during evolution-there are no average imaginary parts to begin with and none are generated during evolution.) A targeted observable on the other hand, like STSA, can give access to the imaginary parts of Wilson line correlators directly. Once a preselected ensemble of events generates such imaginary parts in the average, they also impact the real parts and may even trigger anticorrelations there. The mechanism for this mixing in full JIMWLK lies in the nonlinear nature of the evolution equation. Judging from the agreement between full JIMWLK evolution and the numerical results in the threepoint exponential truncation it would appear that this nonlinear mechanism is well captured in the truncated theory.

The coupling of the odderon component may not have a strong effect on high energy asymptotical behavior even of targeted observables - as we have demonstrated, the odderon still decays with energy even beyond the linear BFKL approximation. At realistic collider energies the question is still open, but only if we consider tailored experiments of sufficient accuracy. If the presence of an odderon contribution comes with anticorrelations in the real parts in its initial condition, this might open new avenues to access them experimentally.

\section{ACKNOWLEDGMENTS}

We are grateful to $H$. Mäntysaari for help with $B K$ numerics. T. L. is supported by the Academy of Finland, Projects No. 267321 and No. 273464. A. R. has been supported by a scholarship from the Center for International Mobility, Finland. K. R. is supported by the Academy of Finland Project No. 267286. H. W. is supported by the National Research Foundation of South Africa (NRF) under CPRR Grant No. 90509. 
[1] H. Weigert, Evolution at small $x_{b j}$ : The color glass condensate, Prog. Part. Nucl. Phys. 55, 461 (2005).

[2] F. Gelis, E. Iancu, J. Jalilian-Marian, and R. Venugopalan, The color glass condensate, Annu. Rev. Nucl. Part. Sci. 60, 463 (2010).

[3] J. Jalilian-Marian, A. Kovner, L. D. McLerran, and H. Weigert, The intrinsic glue distribution at very small $x$, Phys. Rev. D 55, 5414 (1997).

[4] J. Jalilian-Marian, A. Kovner, A. Leonidov, and H. Weigert, The BFKL equation from the Wilson renormalization group, Nucl. Phys. B504, 415 (1997).

[5] J. Jalilian-Marian, A. Kovner, A. Leonidov, and H. Weigert, The Wilson renormalization group for low $x$ physics: Towards the high density regime, Phys. Rev. D 59, 014014 (1998).

[6] J. Jalilian-Marian, A. Kovner, and H. Weigert, The Wilson renormalization group for low $x$ physics: Gluon evolution at finite parton density, Phys. Rev. D 59, 014015 (1998).

[7] J. Jalilian-Marian, A. Kovner, A. Leonidov, and H. Weigert, Unitarization of gluon distribution in the doubly logarithmic regime at high density, Phys. Rev. D 59, 034007 (1999); 59, 099903(E) (1999).

[8] H. Weigert, Unitarity at small Bjorken $x$, Nucl. Phys. A703, 823 (2002).

[9] E. Iancu, A. Leonidov, and L. D. McLerran, Nonlinear gluon evolution in the color glass condensate. I, Nucl. Phys. A692, 583 (2001).

[10] E. Iancu and L. D. McLerran, Saturation and universality in QCD at small $x$, Phys. Lett. B 510, 145 (2001).

[11] E. Ferreiro, E. Iancu, A. Leonidov, and L. McLerran, Nonlinear gluon evolution in the color glass condensate. II, Nucl. Phys. A703, 489 (2002).

[12] E. Iancu, A. Leonidov, and L. D. McLerran, The renormalization group equation for the color glass condensate, Phys. Lett. B 510, 133 (2001).

[13] A.H. Mueller, A simple derivation of the JIMWLK equation, Phys. Lett. B 523, 243 (2001).

[14] I. Balitsky, Operator expansion for high-energy scattering, Nucl. Phys. B463, 99 (1996).

[15] I. Balitsky, Factorization for High-Energy Scattering, Phys. Rev. Lett. 81, 2024 (1998).

[16] I. Balitsky, Factorization and high-energy effective action, Phys. Rev. D 60, 014020 (1999).

[17] I. Balitsky, Effective field theory for the small- $x$ evolution, Phys. Lett. B 518, 235 (2001).

[18] J.-P. Blaizot, E. Iancu, and H. Weigert, Nonlinear gluon evolution in path integral form, Nucl. Phys. A713, 441 (2003).

[19] K. Rummukainen and H. Weigert, Universal features of JIMWLK and BK evolution at small $x$, Nucl. Phys. A739, 183 (2004).

[20] A. Dumitru, J. Jalilian-Marian, T. Lappi, B. Schenke, and R. Venugopalan, Renormalization group evolution of multigluon correlators in high energy QCD, Phys. Lett. B 706, 219 (2011).

[21] T. Lappi and H. Mäntysaari, On the running coupling in the JIMWLK equation, Eur. Phys. J. C 73, 2307 (2013).
[22] Y. V. Kovchegov, Small- $x$ F2 structure function of a nucleus including multiple Pomeron exchanges, Phys. Rev. D 60, 034008 (1999).

[23] L. D. McLerran and R. Venugopalan, Computing quark and gluon distribution functions for very large nuclei, Phys. Rev. D 49, 2233 (1994).

[24] L. D. McLerran and R. Venugopalan, Gluon distribution functions for very large nuclei at small transverse momentum, Phys. Rev. D 49, 3352 (1994).

[25] L. D. McLerran and R. Venugopalan, Green's functions in the color field of a large nucleus, Phys. Rev. D 50, 2225 (1994).

[26] S. Jeon and R. Venugopalan, A classical odderon in QCD at high energies, Phys. Rev. D 71, 125003 (2005).

[27] A. Dumitru, J. Jalilian-Marian, and E. Petreska, Two-gluon correlations and initial conditions for small- $x$ evolution, Phys. Rev. D 84, 014018 (2011).

[28] A. Dumitru and E. Petreska, Initial conditions for dipole evolution beyond the McLerran-Venugopalan model, Nucl. Phys. A879, 59 (2012).

[29] Y. V. Kovchegov, L. Szymanowski, and S. Wallon, Perturbative odderon in the dipole model, Phys. Lett. B 586, 267 (2004).

[30] Y. Hatta, E. Iancu, K. Itakura, and L. McLerran, Odderon in the color glass condensate, Nucl. Phys. A760, 172 (2005).

[31] C. Marquet and H. Weigert, New observables to test the color glass condensate beyond the large- $N_{c}$ limit, Nucl. Phys. A843, 68 (2010).

[32] Y. V. Kovchegov and M. D. Sievert, Sivers function in the quasiclassical approximation, Phys. Rev. D 89, 054035 (2014).

[33] N. Kaiser, Mean eigenvalues for simple, simply connected, compact Lie groups, J. Phys. A 39, 15287 (2006).

[34] K. J. Golec-Biernat and M. Wusthoff, Saturation effects in deep inelastic scattering at low $Q^{2}$ and its implications on diffraction, Phys. Rev. D 59, 014017 (1998).

[35] T. Lappi and H. Mäntysaari, Direct numerical solution of the coordinate space Balitsky-Kovchegov equation at next to leading order, Phys. Rev. D 91, 074016 (2015).

[36] E. Iancu, J. D. Madrigal, A. H. Mueller, G. Soyez, and D. N. Triantafyllopoulos, Resumming double logarithms in the QCD evolution of color dipoles, Phys. Lett. B 744, 293 (2015).

[37] E. Iancu, J. D. Madrigal, A. H. Mueller, G. Soyez, and D. N. Triantafyllopoulos, Collinearly-improved BK evolution meets the HERA data, Phys. Lett. B 750, 643 (2015).

[38] T. Lappi and H. Mäntysaari, Next-to-leading order BalitskyKovchegov equation with resummation, Phys. Rev. D 93, 094004 (2016).

[39] F. Gelis and A. Peshier, Probing colored glass via q anti-q photoproduction, Nucl. Phys. A697, 879 (2002).

[40] J. P. Blaizot, F. Gelis, and R. Venugopalan, High energy pA collisions in the color glass condensate approach I: Gluon production and the Cronin effect, Nucl. Phys. A743, 13 (2004).

[41] J. P. Blaizot, F. Gelis, and R. Venugopalan, High energy pA collisions in the color glass condensate approach II: Quark production, Nucl. Phys. A743, 57 (2004). 
[42] F. Dominguez, C. Marquet, B.-W. Xiao, and F. Yuan, Universality of unintegrated gluon distributions at small $x$, Phys. Rev. D 83, 105005 (2011).

[43] F. Dominguez, C. Marquet, A. M. Stasto, and B.-W. Xiao, Universality of multiparticle production in QCD at high energies, Phys. Rev. D 87, 034007 (2013).

[44] F. Dominguez, C. Marquet, and B. Wu, On multiple scatterings of mesons in hot and cold QCD matter, Nucl. Phys. A823, 99 (2009).

[45] E. Iancu and D. Triantafyllopoulos, JIMWLK evolution in the Gaussian approximation, J. High Energy Phys. 04 (2012) 025.
[46] J. Kuokkanen, K. Rummukainen, and H. Weigert, HERAdata in the light of small $x$ evolution with state of the art NLO input, Nucl. Phys. A875, 29 (2012).

[47] A. Dumitru, T. Lappi, and V. Skokov, The Distribution of Linearly Polarized Gluons and Elliptic Azimuthal Anisotropy in DIS Dijet Production at High Energy, Phys. Rev. Lett. 115, 252301 (2015).

[48] A. Accardi et al., Electron Ion Collider: The next QCD frontier-Understanding the glue that binds us all, arXiv: 1212.1701. 